\title{
Earthquake-induced ground failures in Italy from a reviewed database
}

\author{
S. Martino ${ }^{1}$, A. Prestininzi ${ }^{1}$, and R. W. Romeo ${ }^{2}$ \\ ${ }^{1}$ Sapienza University of Rome, Department of Earth Sciences and Research Centre for Geological Risks (CERI), P.le A. Moro \\ 5, 00185, Rome, Italy \\ ${ }^{2}$ Department of Earth Sciences, Life and Environment, University of Urbino, Italy
}

Correspondence to: S. Martino (salvatore.martino@uniroma1.it)

Received: 30 March 2013 - Published in Nat. Hazards Earth Syst. Sci. Discuss.: 17 May 2013

Revised: 16 February 2014 - Accepted: 24 February 2014 - Published: 10 April 2014

\begin{abstract}
A database (Italian acronym CEDIT) of earthquake-induced ground failures in Italy is presented, and the related content is analysed. The catalogue collects data regarding landslides, liquefaction, ground cracks, surface faulting and ground changes triggered by earthquakes of Mercalli epicentral intensity 8 or greater that occurred in the last millennium in Italy. As of January 2013, the CEDIT database has been available online for public use (http://www.ceri.uniroma1.it/cn/gis.jsp) and is presently hosted by the website of the Research Centre for Geological Risks (CERI) of the Sapienza University of Rome.

Summary statistics of the database content indicate that $14 \%$ of the Italian municipalities have experienced at least one earthquake-induced ground failure and that landslides are the most common ground effects (approximately $45 \%$ ), followed by ground cracks (32\%) and liquefaction (18\%). The relationships between ground effects and earthquake parameters such as seismic source energy (earthquake magnitude and epicentral intensity), local conditions (site intensity) and source-to-site distances are also analysed. The analysis indicates that liquefaction, surface faulting and ground changes are much more dependent on the earthquake source energy (i.e. magnitude) than landslides and ground cracks. In contrast, the latter effects are triggered at lower site intensities and greater epicentral distances than the other environmental effects.
\end{abstract}

\section{Introduction}

The recent strong earthquakes in Sumatra $\left(2004, M_{\mathrm{w}}=9.1\right)$, eastern Sichuan (China 2008, $M_{\mathrm{w}}=7.9$ ) and Tōhoku (Japan 2011, $M_{\mathrm{w}}=9.0$ ) have highlighted that earthquake-induced ground effects (e.g. tsunamis, landslides and liquefaction) can be responsible for major damage and losses, and represent a significant seismic-activity-related hazard (Bird and Bommer, 2004). Such effects can also affect localities tens or hundreds of kilometres distant from the earthquake epicentre, thus increasing the risk related to the earthquake shaking itself (Keefer, 1984; Rodriguez et al., 1999; Delgado et al., 2011; Jibson and Harp, 2012).

In this context, recording and analysing earthquakeinduced ground failures is a relevant contribution to seismic risk mitigation for the purposes of understanding the triggering processes and for identifying areas that might be damaged by future seismic events.

Several studies have been conducted worldwide during the last few decades that report on ground failures triggered by earthquakes (Bommer and Rodriguez, 2002; Sepulveda et al., 2005; Porfido et al., 2007; Tosatti et al., 2008; Gorum et al., 2011; Tang et al., 2011; Alfaro et al., 2012; among many others) and that forecast, using predictive models, the distribution scenarios of earthquake-induced ground effects (Sassa et al., 1996; Jibson et al., 2000; Prestininzi and Romeo, 2000; Romeo, 2000; Jibson, 2007; Hsieh and Lee, 2011; among others). These studies provide inventory maps of the effects that have occurred or susceptibility maps of expected ground failures. Nevertheless, systematic inventories of historically documented earthquake-induced effects have rarely 
been produced until recently. A pioneering study in this field was that by Youd and Hoose (1978) who documented approximately 350 localities affected by ground failures (landslides, lateral spreads, ground settlement and surface cracks) in 46 earthquakes that occurred in Northern California between 1800 and 1970, the most documented one being the devastating 1906 San Francisco $M=8.3$ earthquake. However, until now the majority of information regarding the ground failures triggered by earthquakes is usually not organised as individual catalogues, but included in conventional earthquake catalogues with the purpose to provide further constraints for the assignment of site intensity alone.

The Euro-Mediterranean Earthquake Catalogue (EMEC - Grünthal and Wahlström, 2012) represents the most updated version of the European inventory of earthquakes and related effects, although it does not include a consulting tool for earthquake-induced ground failures or other environmental effects. Similarly, the United States Geological Survey (USGS) composite catalogue PAGER-CAT (Allen et al., 2009), which contains reports of earthquake casualties and losses from the Preliminary Determination of Epicentres (PDE; NEIC, 1970; Sipkin et al., 2000), the Utsu catalogue of deadly earthquakes (Utsu, 2002) and the Emergency Events Database (EM-DAT) developed and maintained by the Centre for Research on the Epidemiology of Disasters at the University of Louvain, Belgium (Hoyois et al., 2007), do not include a direct listing of ground failures even if secondary effects, such as tsunamis, landslides, fires and liquefaction, are also reported in the earthquake record. An original feature of the latter catalogue is that when deaths are caused by these secondary effects, the related information is disaggregated with respect to recorded total deaths.

The current trend is to use the power of the internet to upgrade the existing global databases of environmental effects and to use the collected data to enrich the existing catalogues that were previously created using historical documents or reports. Currently, this modern upgrade process is being applied to several global databases (Petley et al. 2005; Kirschbaum et al., 2010) that are available online and are associated with public-access internet sites that provide map viewer systems linked to geo-databases. Examples include the National Aeronautics and Space Administration (NASA: http://gcmd.nasa.gov/), National Oceanic and Atmospheric Administration (NOAA: http://maps.ngdc.noaa. gov/viewers/) and American Geophysical Union (AGU: http: //blogs.agu.org/blogs/) inventory projects, which are available at their respective web sites.

In addition to the many recent seismic events in Italy that have demonstrated the relevance of ground failures in total earthquake damage (e.g. 1976 Friuli $M_{\mathrm{w}}=6.4 ; 1980$ Irpinia $M_{\mathrm{w}}=6.9 ; 1997$ Umbria-Marche $M_{\mathrm{w}}=6.0 ; 2009$ L'Aquila $M_{\mathrm{w}}=6.2$ ), the last strong Italian earthquake, in May 2012 in Emilia $\left(M_{\mathrm{w}}=6.0\right)$, proved that earthquake-induced ground effects can pose a risk as severe as the earthquake shaking itself (Romeo, 2012).
Over the last decade, many Italian earthquake catalogues containing reported seismic effects (mainly structural and secondarily environmental) have been published online (e.g. CFTI - ING 1995; NT4.1 - Camassi and Stucchi, 1997; DBMI04 - Stucchi et al., 2007; CPTI04 - Gruppo di lavoro CPTI, 2004; ITC 2.0 - Tinti et al., 2007; CPTI11 Rovida et al., 2011). Nevertheless, apart from the tsunamis that are listed in the specific ITC catalogue, other environmental effects cannot be directly found from consulting these catalogues. At the end of the 1990s, Delfino and Romeo (1997) published on the internet the first Italian Catalogue of Earthquake-Induced Ground Failures (the previous release of CEDIT), in which different typologies of ground effects were reported (i.e. landslides, ground cracks, surface faulting, liquefaction and ground changes) over a period of approximately one millennium, from 1000 to 1984 . These effects were further divided into sub-categories, based on landform features or kinematic mechanisms, and information on the involved rocks was also reported. The database structure consisted of tables linked to each other to facilitate consulting and querying, but no visual representation tools were provided.

Presently, the Institute for Environmental Protection and Research (ISPRA) is conducting a project aimed at producing a general catalogue of Earthquake Environmental Effects (EEE: Guerrieri et al., 2009; http://www.eeecatalog.sinanet.apat.it/terremoti/index.php), in which ground effects are categorised into primary effects representing the surface expression of the seismogenic source (e.g. surface faulting, surface uplift and subsidence and any other surface evidence of co-seismic tectonic deformation) and secondary effects (phenomena generally induced by ground shaking), which are classified into the main categories of slope movements, ground settlements, ground cracks, hydrological anomalies, anomalous water waves (including tsunamis) and other effects such as tree shaking, dust clouds, thrown stones, and others. The EEE catalogue is aimed to support the Environmental Seismic Intensity scale (ESI; Michetti et al., 2004, 2007; http://www.isprambiente.gov.it/it/progetti/inqua-scale/ environmental-seismic-intensity-scale-esi-2007), set up to integrate the European Macroseismic Scale (EMS; Grünthal, 1998; http://www.gfz-potsdam.de) that does not consider any environmental effect.

The present paper discusses the new release of the CEDIT catalogue, which revises and updates the previous release (Delfino and Romeo, 1997), and discusses some features of the database content, thus updating the previous study by Prestininzi and Romeo (2000).

\section{Database content and structure}

The new release of the CEDIT database introduces earthquakes and related ground failures that occurred after 1984, 
such as the following: Umbria-Marche $1997\left(M_{\mathrm{w}}=6.0\right)$; Pollino $1998\left(M_{\mathrm{w}}=5.7\right)$; Molise $\left(M_{\mathrm{w}}=5.7\right)$ and the southern Tyrrhenian Sea $\left(M_{\mathrm{w}}=5.9\right)$, both in 2002; L'Aquila 2009 $\left(M_{\mathrm{w}}=6.2\right)$; and Emilia $2012\left(M_{\mathrm{w}}=5.9\right)$. The previous release included all bibliographic sources available for historical earthquakes. However, new studies that have retrieved information about some historical earthquakes, published after the first release of the catalogue, made it necessary to revise the related data. Reference was made particularly to papers referring to specific earthquakes, such as those by Porfido et al. (2007) and Serva et al. (2007) that detail the ground effects that occurred in some earthquakes in historical (Sannio 1805) and recent times (Irpinia 1930 and 1980); studies referring to specific ground phenomena, such as the catalogue of liquefaction by Galli (2000); and studies referring to specific localities where earthquakes have produced some outstanding effect (Prestininzi, 1995; Mancini et al., 2001; Martino et al., 2004; Bozzano et al., 2004, 2008, 2011).

The CEDIT database reports approximately 2000 localities where ground failures were triggered by 166 earthquakes that occurred in the last millennium in Italy for which information about the occurrence of ground effects can be retrieved from historical documents. It is worth mentioning that only the name of the localities and a generic description of the type of ground failures can be reliably retrieved from the historical sources of information, especially when they refer to very old earthquakes. Therefore, a locality affected by several ground failures is counted only once, since in many cases there is no information about the number of triggered failures.

The ground effects collected in the database fall into five main categories: landslides, ground cracks, liquefaction, surface faulting and ground changes (these last ones including among others: subsidence, relevant morphological changes due to river damming, lake formation and so on). These categories are further divided into sub-categories, specifying the type of effect, such as, for example, the landslide kinematic type. The database underlying the new release of CEDIT is organised into data sheets that contain data about earthquakes and their associated ground failures (Fortunato et al., 2012). The relational database (Fig. 1) consists of five tables: "TERREMOTI" (the Italian translation of earthquakes), "LOCALITÀ" (localities), "FRASI" (sentences), "BIBLIO" (references), and "EFFETTI" (ground failures).

Data were collected from different seismic catalogues, as shown in Fig. 1, including the CPTI04 catalogue of earthquakes (Gruppo di lavoro CPTI, 2004), the DBMI04 macroseismic catalogue (Stucchi et al., 2007), the Catalogue of Italian Tsunamis (ITC 2.0; Tinti et al., 2007) and the Database of the Italian Seismogenic Sources (DISS 3.1.1; DISS WORKING GROUP, 2010). Localities where ground failures occurred were mapped according to the WGS 84 coordinates system and are identified by an administrative code assigned by the Central Institute of Statistics (ISTAT; www.istat.it/ en/).

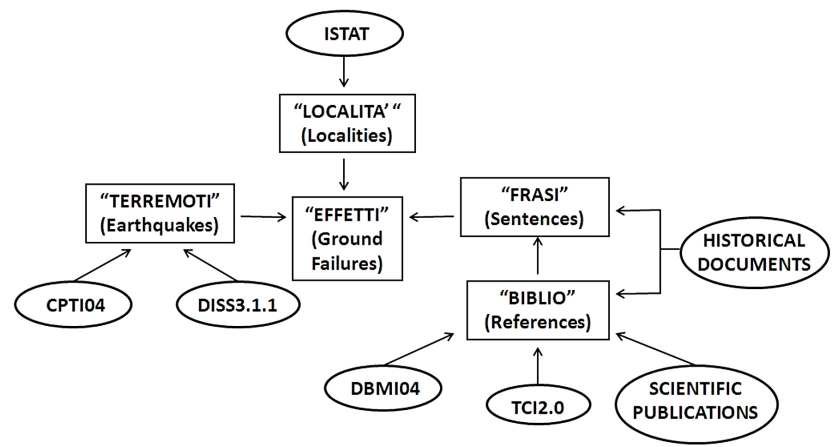

Fig. 1. Database structure of the CEDIT catalogue and links to the CPTI04 earthquake catalogue (Gruppo di lavoro CPTI, 2004), the DBMI04 macroseismic catalogue (Stucchi et al., 2007), the ITC 2.0 Italian tsunami catalogue (Tinti et al., 2007) and the DISS 3.1.1 seismic sources database (DISS Working Group, 2010).

The original descriptions gathered from the historical sources that describe the ground failures are preserved to allow for the retrieval of each effect. Moreover, to provide as much detailed information on each effect as possible, quotations from various authors about the same effect are also reported. For an improved understanding of the extent of seismically induced effects, the values of the dimensional parameters reported in the historical documents regarding distances, volumes and masses were converted into the decimal metric system, a process based on specific studies on the conversion between historical and modern measurement systems (Martini, 1883).

The CEDIT database is published online for public access at http://www.ceri.uniroma1.it/cn/gis.jsp and is hosted by the web server of the Research Centre for the Geological Risks (CERI) of the Sapienza University of Rome (Fortunato et al., 2012). The query system was developed by using the services of ArcGIS ${ }^{\circledR}$-online and based on ESRI ${ }^{\mathrm{TM}}$ cloud technology. The system provides a geo-database consulting and querying interface with graph or table outputs.

\section{Descriptive statistics}

The current version is an upgrade of the first release of the CEDIT database (Romeo and Delfino, 1997). Figure 2 shows a comparison between the two versions of the catalogue.

In the new release, the number of recorded landslides has increased compared with the first release due to the huge effort of the Italian scientific community in recent years to retrieve as much information and data as possible regarding landslides and other mass movements as part of an attempt to reduce the geologic hazards of the country. Similarly, another effect extensively investigated in recent years has been surface faulting, for which the number of records has been greatly increased in the new database. Although surface faulting is a primary effect of fault rupture, whereas ground 

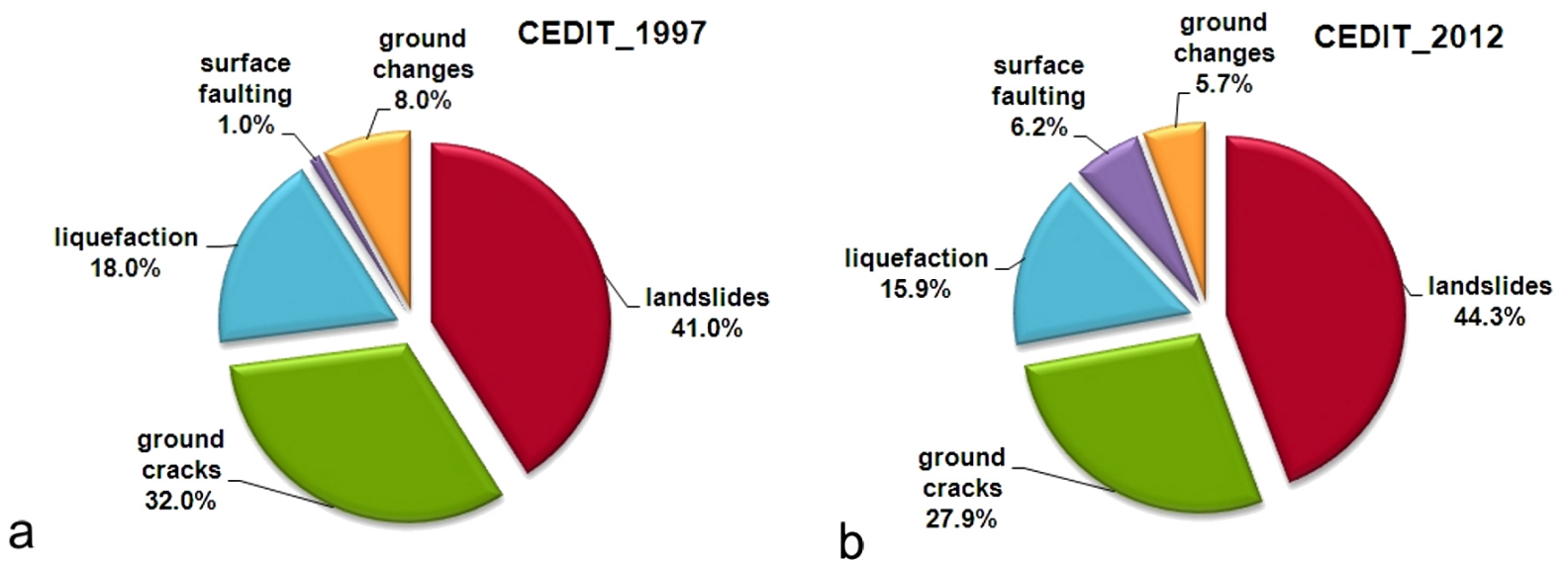

Fig. 2. Percentage of ground failures included (a) in the previous release of the CEDIT database (until 1984) and (b) in the current version of the CEDIT database (until 2012).

failures are secondary effects resulting from the ground response to the seismic shaking, in the current version of the database this effect has been retained due to its primary importance for the identification of active faults and the implications for seismic hazard (e.g. surface faulting is a primary cause of site rejection for many structures, especially in the case of critical facilities such as power plants and dams).

Among landslides, approximately $40 \%$ can be ascribed to Keefer's $(1984,2002)$ type-1 category of landslides triggered by earthquakes (falls and disrupted slides), $22 \%$ to type-2 (coherent slides), $6 \%$ to type-3 (lateral spreads and flows) and a considerable number (approximately 32\%) are undefined. Type- 3 landslides were distinguished from liquefaction-related effects based on the description given in the historical chronicles, which can be retrieved in the online version of the catalogue. For instance, in the case of slopes where the clear news of water ejected from the ground was not documented, the ground effect was interpreted as a landslide, otherwise as a liquefaction-related effect.

Figure 3 shows the time distribution of the earthquakes reported in the CEDIT database from which data about ground failures have been gathered.

Despite the general tendency of earthquakes towards clustering, the time distribution is clearly more continuous starting from the end of the 18th century as a consequence of the seismic crisis that affected the Calabria region in 1783. The crisis altered the way earthquakes and their effects were detected, as this event marked the first time in Italy (and perhaps in the world; Keefer, 2002) that a scientific mission was launched to detect and report earthquake damage and collateral hazards (Sarconi, 1784). This event also represented the first example of seismic technical provisions given by the local authorities for the reconstruction of the villages damaged by earthquake sequences (Postpischl, 1985a, b). Figure 4 shows the time distribution of the environmental effects reported in the CEDIT database.

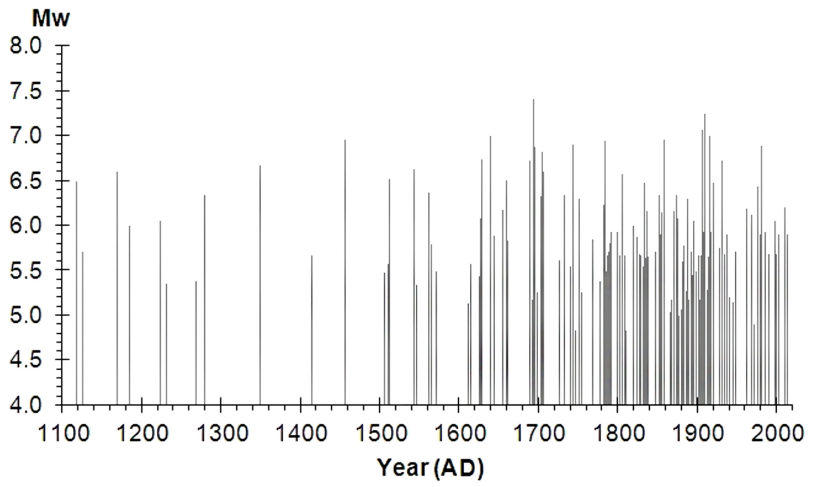

Fig. 3. Time distribution of earthquake magnitudes reported in the CEDIT database. A considerable increase of earthquakes from which information about localities affected by ground failures have been retrieved can be observed since the 19th century, after the Calabria, 1783 seismic sequence that marked the start up of scientific technical surveys after an earthquake occurrence.

Two major increases in the cumulative number of ground failures are apparent: the first increase relates to the already cited earthquake sequence that struck the Calabria region (Southern Italy) in 1783. This sequence involved at least three major earthquakes above magnitude 6.5 that triggered several ground failures, 145 of which are reported in the catalogue (Vivenzio, 1788; Minasi, 1785; De Lorenzo, 1877; Graziani et al., 2006). The second increase relates to the 1976 Friuli $M_{\mathrm{w}}=6.4$ earthquake (northern Italy). This earthquake represents for Italy the starting point of the systematic development and detection of strong motion records, damage and environmental effects (Carraro et al., 1976). Moreover, since 1970s the availability of aerial surveys has allowed the detection of a number of effects more complete than in the past. Two other dates are worthy of mentioning: the first is the beginning of the last century which represents the starting of 


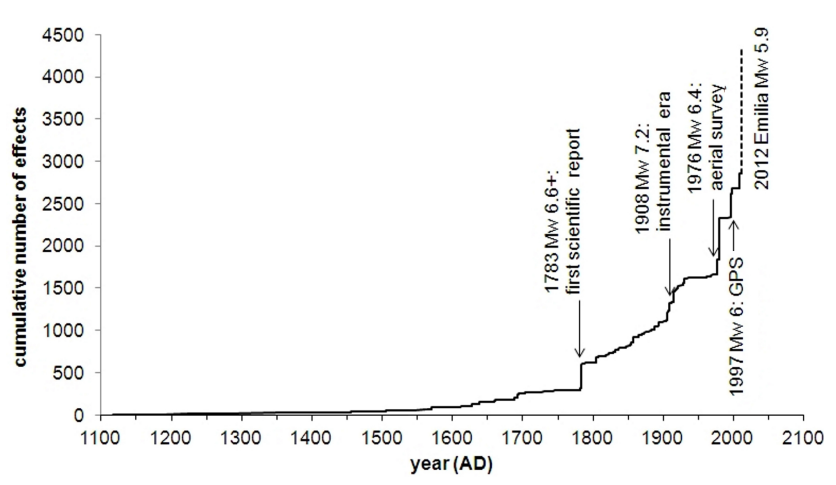

Fig. 4. Time distribution of the cumulative number of ground failures reported in the CEDIT database. Marked dates refer to turning points in the completeness and reliability of detected localities and effects, i.e., from older to younger: 1783 Calabrie $M_{\mathrm{W}}=6.6+$ earthquakes, first scientific report; 1908 Messina Strait $M_{\mathrm{W}}=7.2$ earthquake, beginning of the seismological instrumental era; 1976 Friuli $M_{\mathrm{W}}=6.4$ earthquake, systematic aerial surveys; 1997 Umbria-Marche $M_{\mathrm{W}}=6$ seismic sequence, starting point of GPS measurements; 2012 Emilia $M_{\mathrm{W}}=5.9$ seismic sequence, most recent event.

the instrumental age from the seismological point of view. In fact, since the 1908 Stretto di Messina earthquake, the availability of instrumentally detected epicentres and magnitudes has allowed increasing the accuracy of earthquakes' parameters, whose sources of error were an unavoidable component in all the previous earthquakes. The second date is starting from the 1990s, when the diffusion of GPS measurements has virtually eliminated the error in the ground effects location. However, despite these key dates the catalogue is far from being complete, considering that most ground failures occur in scarcely populated areas, such as mountainous regions; even for the most recent events, the detection of such effects can be incomplete because the survey is often influenced by the induced risk (e.g. damage to buildings, lifelines and infrastructures), thus missing the detection of small or non-damaging ground effects.

As far the completeness of the earthquake magnitude records are concerned, in Fig. 5, the number of earthquakes reported in the CEDIT database is compared with the number of earthquakes listed in the CPTI earthquake catalogue.

The CPTI catalogue lists the earthquakes that occurred in Italy in the last millennium that are significant for seismic hazard assessments. The threshold of completeness of the CPTI catalogue is between $M_{\mathrm{w}}=4.5$ and 5.0, after which there is a rapid decrease in the number of earthquakes per magnitude class that clearly follows an exponential decay pattern. The modal magnitude of the CEDIT database is between 5.5 and 6.0, which roughly corresponds to epicentral intensity 8 in the Mercalli scale, as further shown in Fig. 6. The decay of the number of earthquakes per magnitude class in the CEDIT database is less pronounced than in the CPTI catalogue because the larger magnitudes are associated with

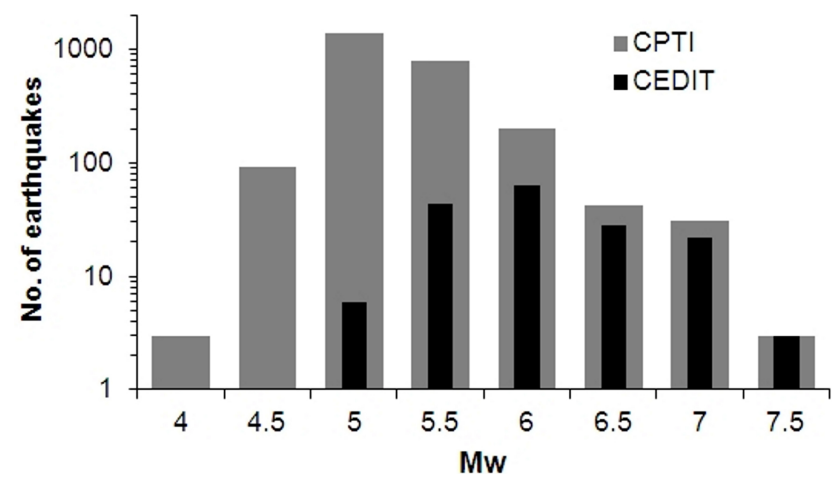

Fig. 5. Number of earthquakes per magnitude class reported in the CPTI earthquake catalogue for seismic hazard analyses and in the CEDIT database. In each graph of this paper showing on the $x$ axis an interval scale, the interval itself is defined as the upper limit included and the lower limit excluded, i.e. $x_{i-1}<X \leq x_{i}$.

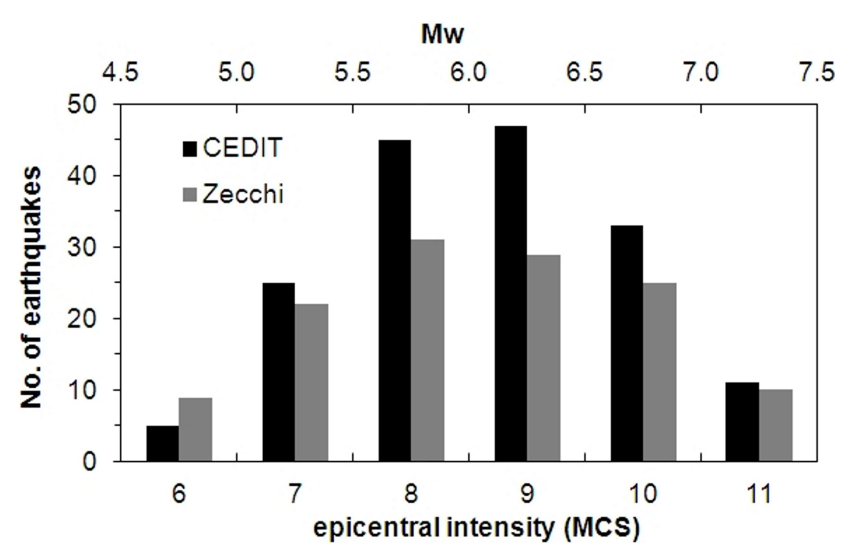

Fig. 6. Distribution of earthquakes per epicentral intensity that triggered ground failures in Italy as reported on the map by Zecchi (1987) and in the CEDIT database. The upper $x$ axis shows an approximate conversion of epicentral intensity into moment magnitude (from Gruppo di Lavoro CPTI, 2004, with small adjustments).

a higher likelihood of ground-failure triggering. Only approximately $15 \%$ of the earthquakes above magnitude 5 of the CPTI catalogue are included in the CEDIT database, because most of the earthquakes listed in the CPTI occurred in historical times, when the attention mainly focused on building damage rather than on environmental damage. The list of earthquakes for which ground effects have been derived from the historical data sources are reported in Table 1, along with the number of affected localities and the inferred ground effects. For the earthquakes since 1900 (instrumental age), the focal depth, when available, is reported, too. Whereas hypocentral depth can play an important role in determining the ground response, almost all the earthquakes listed in Table 1 are reasonably crustal earthquakes (depth less than $30 \mathrm{~km}$ ), according to the seismotectonic framework of Italy (Meletti et al., 2000). As an example of the possible influence of hypocentral depth, in the Emilia 2012 seismic sequence 
Table 1. List of earthquake records in the CEDIT database with affected localities and triggered ground failures.

\begin{tabular}{|c|c|c|c|c|c|c|c|c|c|c|c|c|c|}
\hline $\begin{array}{l}\# \\
\text { eqk }\end{array}$ & $\begin{array}{l}\text { ID } \\
\text { CPTI04 }\end{array}$ & $\begin{array}{l}\text { Epicentral } \\
\text { area }\end{array}$ & Date & Lat $^{\circ}$ & Long $^{\circ}$ & Io(MCS) & $\begin{array}{l}M_{\mathrm{W}} \\
\text { (depth) }\end{array}$ & Sites & $\begin{array}{l}\text { Land- } \\
\text { slides }\end{array}$ & $\begin{array}{l}\text { Ground } \\
\text { cracks }\end{array}$ & $\begin{array}{l}\text { Lique- } \\
\text { faction }\end{array}$ & $\begin{array}{l}\text { Surface } \\
\text { faulting }\end{array}$ & $\begin{array}{l}\text { Ground } \\
\text { changes }\end{array}$ \\
\hline 1 & 30 & Veronese & $1117-01-03$ & 45.330 & 11.200 & 9.5 & 6.49 & 2 & & & 1 & & 1 \\
\hline 2 & 33 & Sannio-Molise & $1125-10-11$ & 41.600 & 15.000 & 8.0 & 5.71 & 1 & & 1 & & & \\
\hline 3 & 37 & Sicilia orientale & $1169-02-04$ & 37.320 & 15.030 & 10.0 & 6.60 & 3 & 1 & 1 & 3 & & \\
\hline 4 & 40 & Valle del Crati & $1184-05-24$ & 39.430 & 16.250 & 9.0 & 6.00 & 1 & 1 & & & & \\
\hline 5 & 47 & Basso Bresciano & $1222-12-25$ & 45.480 & 10.680 & 8.5 & 6.05 & 5 & 4 & 2 & & & \\
\hline 6 & 50 & Cassino & 1231-06-01 & 41.480 & 13.830 & 7.0 & 5.35 & 1 & 2 & 1 & & & \\
\hline 7 & 55 & Trevigiano & $1268-11-04$ & 45.730 & 12.080 & 7.5 & 5.37 & 1 & 1 & & & & \\
\hline 8 & 63 & Camerino & $1279-04-30$ & 43.093 & 12.872 & 10.0 & 6.33 & 1 & 1 & & & & \\
\hline 9 & 94 & Carnia & $1348-01-25$ & 46.254 & 12.883 & 9.5 & 6.66 & 2 & 1 & 2 & 1 & & \\
\hline 10 & 95 & Lazio meridionale-Molise & 1349-09-09 & 41.480 & 14.070 & 10.0 & 6.62 & 3 & 2 & 1 & & & \\
\hline 11 & 135 & Toscana occidentale & $1414-08-07$ & 43.271 & 11.120 & 7.5 & 5.66 & 1 & & 1 & & & \\
\hline 12 & 153 & Molise & 1456-12-05 & 41.302 & 14.711 & 10.0 & 6.96 & 13 & 4 & 8 & & & 3 \\
\hline 13 & 202 & Bologna & $1505-01-03$ & 44.480 & 11.250 & 7.0 & 5.47 & 2 & 1 & 1 & 2 & & \\
\hline 14 & 208 & Calabria meridionale & $1509-02-25$ & 38.100 & 15.680 & 8.0 & 5.57 & 1 & 1 & 1 & & & \\
\hline 15 & 210 & Slovenia & $1511-03-26$ & 46.200 & 13.430 & 9.0 & 6.51 & 1 & 1 & & & & \\
\hline 16 & 238 & Mugello & $1542-06-13$ & 44.000 & 11.380 & 9.0 & 5.91 & 1 & & & 1 & & \\
\hline 17 & 240 & Siracusano & $1542-12-10$ & 37.220 & 14.950 & 10.0 & 6.62 & 1 & & & 2 & & \\
\hline 18 & 241 & Borgo Val di Taro & $1545-06-09$ & 44.498 & 9.844 & 7.5 & 5.33 & 4 & 2 & 1 & 1 & & 2 \\
\hline 19 & 256 & Vallo di Diano & 1561-08-19 & 40.520 & 15.480 & 9.5 & 6.36 & 6 & 3 & 3 & 2 & & \\
\hline 20 & 259 & Alpi marittime & $1564-07-20$ & 44.022 & 7.278 & 8.5 & 5.79 & 2 & 1 & 1 & & & \\
\hline 21 & 262 & Ferrara & $1570-11-17$ & 44.820 & 11.630 & 7.5 & 5.48 & 10 & 1 & 8 & 14 & & 4 \\
\hline 22 & 308 & Scarperia & $1611-09-08$ & 44.020 & 11.370 & 7.0 & 5.13 & 1 & & 1 & & & \\
\hline 23 & 311 & Naso & $1613-08-25$ & 38.120 & 14.780 & 8.0 & 5.57 & 1 & 1 & 2 & & & \\
\hline 24 & 323 & Argenta & $1624-03-18$ & 44.650 & 11.850 & 7.5 & 5.43 & 2 & & 1 & 3 & & 1 \\
\hline 25 & 327 & Girifalco & $1626-04-04$ & 38.820 & 16.420 & 9.0 & 6.08 & 2 & & 2 & & & \\
\hline 26 & 330 & Gargano & $1627-07-30$ & 41.730 & 15.350 & 10.0 & 6.73 & 15 & 5 & 8 & 10 & & 2 \\
\hline 27 & 341 & Calabria & $1638-03-27$ & 39.030 & 16.280 & 11.0 & 7.00 & 15 & 4 & 11 & 4 & 1 & 2 \\
\hline 28 & 343 & Amatrice & 1639-10-07 & 42.636 & 13.252 & 10.0 & 6.26 & 1 & & & & & 1 \\
\hline 29 & 349 & Alpi marittime & $1644-02-15$ & 43.980 & 7.320 & 8.5 & 5.88 & 1 & 1 & & & & \\
\hline 30 & 358 & Sorano-Marsica & $1654-07-23$ & 41.630 & 13.680 & 9.5 & 6.17 & 3 & 1 & 2 & & & \\
\hline 31 & 361 & Calabria centrale & $1659-11-05$ & 38.700 & 16.250 & 10.0 & 6.50 & 6 & 2 & 5 & & & \\
\hline 32 & 365 & Montecchio & $1661-03-12$ & 45.730 & 10.070 & 7.0 & 5.17 & 4 & 4 & 2 & & & \\
\hline 33 & 366 & Appennino Romagnolo & $1661-03-22$ & 44.020 & 11.900 & 9.0 & 5.83 & 6 & & 5 & 1 & & \\
\hline 34 & 393 & Romagna & $1688-04-11$ & 44.390 & 11.942 & 9.0 & 5.88 & 2 & 2 & 1 & & & 1 \\
\hline 35 & 394 & Sannio & 1688-06-05 & 41.280 & 14.570 & 11.0 & 6.72 & 14 & 4 & 9 & 4 & & 1 \\
\hline 36 & 407 & M. Valcalda & $1692-05-00$ & 46.350 & 12.800 & 7.0 & 5.17 & 1 & 1 & & & & \\
\hline 37 & 410 & Sicilia orientale & 1693-01-11 & 37.130 & 15.020 & 11.0 & 7.41 & 20 & 7 & 11 & 16 & & 4 \\
\hline 38 & 414 & Irpinia-Basilicata & 1694-09-08 & 40.880 & 15.350 & 10.5 & 6.87 & 12 & 5 & 9 & & & \\
\hline 39 & 417 & Bagnoregio & $1695-06-11$ & 42.612 & 12.110 & 8.5 & 5.77 & 1 & 1 & 1 & & & \\
\hline 40 & 424 & Vizzini & $1698-04-12$ & 37.312 & 14.878 & 7.0 & 5.25 & 1 & 1 & & & & \\
\hline 41 & 430 & Beneventano-Irpinia & 1702-03-14 & 41.120 & 14.980 & 9.5 & 6.32 & 1 & 1 & & & & \\
\hline 42 & 434 & Appennino reatino & $1703-01-14$ & 42.680 & 13.120 & 11.0 & 6.81 & 5 & 2 & 3 & & & \\
\hline 43 & 435 & Aquilano & $1703-02-02$ & 42.470 & 13.200 & 10.0 & 6.65 & 2 & & & 3 & & \\
\hline 44 & 439 & Villafranca & $1703-12-28$ & 44.780 & 7.505 & 7.5 & 5.37 & 1 & 1 & & & & \\
\hline 45 & 445 & Maiella & $1706-11-03$ & 42.080 & 14.080 & 9.5 & 6.60 & 5 & 2 & 3 & & & \\
\hline 46 & 484 & Palermo & $1726-09-01$ & 38.120 & 13.350 & 8.0 & 5.61 & 2 & 1 & 1 & & & \\
\hline 47 & 496 & Foggiano & $1731-03-20$ & 41.270 & 15.750 & 9.0 & 6.34 & 1 & & & 3 & & \\
\hline 48 & 513 & Naso & $1739-05-10$ & 38.100 & 14.750 & 8.0 & 5.54 & 4 & 2 & 3 & & & 1 \\
\hline 49 & 520 & Basso Ionio & $1743-02-20$ & 39.850 & 18.780 & 9.5 & 6.90 & 2 & & 2 & & & \\
\hline 50 & 526 & Garfagnana & $1746-07-23$ & 44.088 & 10.444 & 6.0 & 4.83 & 2 & 3 & & & & \\
\hline 51 & 535 & Gualdo Tadino & 1751-07-27 & 43.222 & 12.730 & 10.0 & 6.30 & 1 & 1 & & & & \\
\hline 52 & 540 & Valle del Chisone & $1753-03-09$ & 44.930 & 7.180 & 6.5 & 5.25 & 3 & 1 & 2 & 2 & & \\
\hline 53 & 575 & Appennino Romagnolo & $1768-10-19$ & 43.930 & 11.870 & 9.0 & 5.84 & 1 & & 1 & & & \\
\hline 54 & 601 & Radicofani & $1777-10-05$ & 42.880 & 11.756 & 7.5 & 5.37 & 1 & 1 & & & & \\
\hline 55 & 616 & Faentino & $1781-04-04$ & 44.235 & 11.797 & 9.0 & 5.84 & 4 & & 4 & 5 & & \\
\hline 56 & 618 & Romagna & 1781-06-03 & 43.594 & 12.506 & 9.5 & 6.23 & 5 & 5 & 4 & & & \\
\hline 57 & 619 & Cagliese & $1781-07-17$ & 44.280 & 11.950 & 8.0 & 5.53 & 1 & & & & & 1 \\
\hline 58 & 626 & Calabria & $1783-02-05$ & 38.300 & 15.970 & 11.0 & 6.91 & 98 & 58 & 47 & 70 & & 32 \\
\hline 59 & 628 & Calabria & 1783-02-07 & 38.580 & 16.200 & 10.5 & 6.59 & 20 & 11 & 8 & 6 & & 4 \\
\hline 60 & 629 & Calabria & $1783-03-01$ & 38.770 & 16.300 & 9.0 & 5.92 & 1 & & 1 & & & \\
\hline 61 & 630 & Calabria centrale & $1783-03-28$ & 38.780 & 16.470 & 10.0 & 6.94 & 26 & 10 & 13 & 23 & & 3 \\
\hline 62 & 637 & Gerace & $1784-10-14$ & 38.293 & 16.210 & 7.0 & 5.09 & 1 & 1 & & & & \\
\hline 63 & 643 & Piediluco & 1785-10-09 & 42.564 & 12.777 & 8.0 & 5.48 & 2 & & 1 & 3 & & \\
\hline 64 & 651 & Riminese & $1786-12-25$ & 43.980 & 12.580 & 8.0 & 5.67 & 1 & 1 & 1 & 1 & & \\
\hline 65 & 661 & Tolmezzo & $1788-10-20$ & 46.398 & 13.019 & 8.5 & 5.71 & 1 & & 1 & & & 1 \\
\hline 66 & 663 & Val Tiberina & $1789-09-30$ & 43.505 & 12.208 & 8.5 & 5.80 & 1 & & & 2 & & 1 \\
\hline 67 & 667 & Scopoli & $1791-10-11$ & 42.972 & 12.824 & 7.5 & 5.32 & 1 & & 1 & & & \\
\hline
\end{tabular}


Table 1. Continued.

\begin{tabular}{|c|c|c|c|c|c|c|c|c|c|c|c|c|c|}
\hline $\begin{array}{l}\# \\
\text { eqk }\end{array}$ & $\begin{array}{l}\text { ID } \\
\text { CPTI04 }\end{array}$ & $\begin{array}{l}\text { Epicentral } \\
\text { area }\end{array}$ & Date & Lat $^{\circ}$ & Long $^{\circ}$ & Io(MCS) & $\begin{array}{l}M_{\mathrm{W}} \\
\text { (depth) }\end{array}$ & Sites & $\begin{array}{l}\text { Land- } \\
\text { slides }\end{array}$ & $\begin{array}{l}\text { Ground } \\
\text { cracks }\end{array}$ & $\begin{array}{l}\text { Lique- } \\
\text { faction }\end{array}$ & $\begin{array}{l}\text { Surface } \\
\text { faulting }\end{array}$ & $\begin{array}{l}\text { Ground } \\
\text { changes }\end{array}$ \\
\hline 68 & 668 & Calabria centrale & $1791-10-13$ & 38.630 & 16.270 & 9.0 & 5.92 & 1 & & 1 & & & \\
\hline 69 & 687 & Camerino & $1799-07-28$ & 43.147 & 13.123 & 9.0 & 5.93 & 2 & & 1 & & & 1 \\
\hline 70 & 694 & Valle dell'Oglio & $1802-05-12$ & 45.420 & 9.850 & 8.0 & 5.67 & 3 & & 2 & 2 & & \\
\hline 71 & 700 & Molise & $1805-07-26$ & 41.500 & 14.470 & 10.0 & 6.57 & 42 & 32 & 19 & 6 & 4 & 2 \\
\hline 72 & 710 & Valle del Pellice & 1808-04-02 & 44.830 & 7.250 & 8.0 & 5.67 & 9 & 2 & 8 & & & \\
\hline 73 & 714 & Malcesine & $1810-05-01$ & 45.764 & 10.809 & 6.0 & 4.83 & 1 & & 1 & & & \\
\hline 74 & 736 & Catanese & $1818-02-20$ & 37.600 & 15.130 & 9.0 & 6.00 & 9 & & 7 & 6 & & \\
\hline 75 & 739 & Madonie & $1818-09-08$ & 37.820 & 14.080 & 7.5 & 5.31 & 3 & 3 & 2 & & & \\
\hline 76 & 752 & Sicilia settentrionale & $1823-03-05$ & 38.000 & 14.100 & 8.5 & 5.87 & 9 & 3 & 7 & 2 & & 2 \\
\hline 77 & 759 & Basilicata & $1826-02-01$ & 40.520 & 15.730 & 8.0 & 5.68 & 2 & 2 & & 2 & & \\
\hline 78 & 770 & Valle dello Staffora & $1828-02-02$ & 40.750 & 13.900 & 8.0 & 5.57 & 1 & 1 & & & & \\
\hline 79 & 776 & Casamicciola Terme & $1828-10-09$ & 44.820 & 9.050 & 7.5 & 5.67 & 7 & 7 & & & & \\
\hline 80 & 790 & Liguria occidentale & $1831-05-26$ & 43.850 & 7.850 & 8.0 & 5.54 & 2 & 2 & 1 & & & \\
\hline 81 & 795 & Reggiano & $1832-01-13$ & 42.967 & 12.659 & 8.5 & 5.80 & 5 & & 3 & 5 & & \\
\hline 82 & 797 & Foligno & $1832-03-08$ & 39.070 & 16.900 & 9.5 & 6.48 & 7 & 3 & 4 & 7 & & \\
\hline 83 & 798 & Crotonese & $1832-03-13$ & 44.770 & 10.470 & 7.5 & 5.59 & 3 & & 1 & 1 & & 1 \\
\hline 84 & 801 & Alta Lunigiana & $1834-02-14$ & 44.449 & 9.859 & 8.5 & 5.64 & 1 & 1 & 1 & & & 1 \\
\hline 85 & 808 & Cosentino & $1835-10-12$ & 39.330 & 16.300 & 9.0 & 5.91 & 2 & 3 & 1 & & & \\
\hline 86 & 811 & Basilicata meridionale & $1836-04-25$ & 39.570 & 16.730 & 9.0 & 6.16 & 4 & 1 & 3 & 3 & & 2 \\
\hline 87 & 815 & Calabria settentrionale & $1836-11-20$ & 40.150 & 15.780 & 8.0 & 5.83 & 2 & 2 & 2 & & & \\
\hline 88 & 819 & Alpi Apuane & $1837-04-11$ & 44.174 & 10.181 & 9.5 & 5.65 & 1 & 1 & 1 & & & 1 \\
\hline 89 & 855 & Orciano Pisano & $1846-08-14$ & 43.531 & 10.500 & 8.5 & 5.71 & 11 & 3 & 5 & 14 & & \\
\hline 90 & 878 & Basilicata & 1851-08-14 & 40.950 & 15.670 & 9.5 & 6.33 & 6 & 7 & 3 & & & \\
\hline 91 & 886 & Moggio Udinese & $1853-02-19$ & 46.383 & 13.100 & 7.0 & 5.17 & 1 & 1 & & & & \\
\hline 92 & 887 & Irpinia & 1853-04-09 & 40.820 & 15.220 & 9.0 & 5.90 & 3 & 2 & 2 & & & \\
\hline 93 & 893 & Cosentino & $1854-02-12$ & 39.250 & 16.300 & 9.5 & 6.15 & 16 & 11 & 14 & 4 & & \\
\hline 94 & 899 & Vallese & $1855-07-25$ & 46.217 & 7.850 & 8.5 & 5.81 & 4 & 3 & & 1 & & \\
\hline 95 & 912 & Basilicata & $1857-12-16$ & 40.350 & 15.850 & 10.5 & 6.96 & 35 & 32 & 21 & 2 & 1 & 2 \\
\hline 96 & 945 & Area etnea & $1865-07-19$ & 37.700 & 15.150 & 9.0 & 5.03 & 15 & 5 & 13 & & 6 & \\
\hline 97 & 950 & Monte Baldo & $1866-08-11$ & 45.727 & 10.783 & 7.0 & 5.17 & 1 & 1 & & & & \\
\hline 98 & 970 & Cosentino & $1870-10-04$ & 39.220 & 16.330 & 9.5 & 6.16 & 7 & 4 & 3 & 3 & & \\
\hline 99 & 985 & Bellunese & $1873-06-29$ & 46.150 & 12.380 & 9.5 & 6.33 & 16 & 9 & 13 & 1 & & \\
\hline 100 & 1000 & Romagna sud-orientale & $1875-03-17$ & 44.070 & 12.550 & 8.0 & 5.74 & 2 & & 2 & 2 & & \\
\hline 101 & 1003 & San Marco in Lamis & $1875-12-06$ & 41.689 & 15.677 & 7.5 & 6.07 & 1 & & & 1 & & \\
\hline 102 & 1005 & Monte Baldo & $1876-04-29$ & 45.750 & 10.780 & 7.0 & 4.99 & 1 & 1 & 1 & & & \\
\hline 103 & 1043 & Area etnea & 1879-06-17 & 37.680 & 15.150 & 9.0 & 5.06 & 6 & 4 & 2 & & & \\
\hline 104 & 1066 & Abruzzo Meridionale & 1881-09-10 & 42.230 & 14.280 & 8.0 & 5.59 & 1 & 1 & 1 & & & 1 \\
\hline 105 & 1082 & Monte Baldo & $1882-09-18$ & 45.720 & 10.770 & 7.0 & 5.17 & 1 & 1 & & & & \\
\hline 106 & 1088 & Casamicciola Terme & $1883-07-28$ & 40.750 & 13.880 & 9.0 & 5.78 & 9 & 7 & 4 & & & \\
\hline 107 & 1111 & Benevento & $1885-09-17$ & 41.133 & 14.800 & 7.0 & 5.17 & 1 & & 1 & & & \\
\hline 108 & 1121 & Val di Susa & 1886-09-05 & 45.036 & 7.306 & 6.5 & 5.27 & 1 & 1 & & & & \\
\hline 109 & 1128 & Liguria occidentale & $1887-02-23$ & 43.920 & 8.070 & 9.0 & 6.29 & 25 & 9 & 15 & 6 & & 2 \\
\hline 110 & 1136 & Calabria settentrionale & $1887-12-03$ & 39.570 & 16.220 & 8.0 & 5.52 & 3 & 3 & & & & \\
\hline 111 & 1154 & Tolmezzo & $1889-10-13$ & 46.400 & 13.000 & 7.0 & 5.17 & 1 & & 1 & & & \\
\hline 112 & 1170 & Valle d'Illasi & 1891-06-07 & 45.570 & 11.170 & 8.5 & 5.71 & 7 & 5 & 2 & & & \\
\hline 113 & 1186 & Pont Saint Martin & $1892-03-05$ & 45.569 & 7.797 & 7.0 & 5.09 & 2 & 2 & & & & \\
\hline 114 & 1207 & Gargano & $1893-08-10$ & 41.720 & 16.080 & 8.0 & 5.44 & 2 & 2 & 2 & & & \\
\hline 115 & 1212 & Lesina & $1894-03-25$ & 41.867 & 15.323 & 7.0 & 5.17 & 1 & & 1 & & & \\
\hline 116 & 1215 & Calabria meridionale & 1894-08-08 & 37.650 & 15.120 & 9.5 & 5.23 & 5 & 3 & 4 & & & \\
\hline 117 & 1216 & Area etnea & 1894-11-16 & 38.280 & 15.870 & 8.5 & 6.05 & 24 & 10 & 17 & 3 & & 5 \\
\hline 118 & 1229 & Bajano & $1895-05-20$ & 42.750 & 12.700 & 7.0 & 5.17 & 1 & & 1 & & & \\
\hline 119 & 1291 & Calestano & 1898-03-04 & 44.503 & 10.314 & 6.5 & 5.07 & 2 & 1 & & 1 & & \\
\hline 120 & 1299 & Rieti & $1898-06-27$ & 42.415 & 12.905 & 7.5 & 5.48 & 1 & 1 & & & & \\
\hline 121 & 1304 & Caltagirone & 1898-11-02 & 37.216 & 14.495 & 5.5 & 4.63 & 1 & & & 1 & & \\
\hline 122 & 1340 & Salò & 1901-03-29 & 45.167 & 7.167 & 6.0 & 4.63 & 1 & & & 1 & & \\
\hline 123 & 1342 & M. Lera & $1901-04-24$ & 42.100 & 12.736 & 7.5 & 5.15 & 1 & & 1 & 1 & & \\
\hline 124 & 1353 & Montelibretti & $1901-10-30$ & 45.580 & 10.500 & 8.0 & 5.67 & 2 & 2 & 1 & & & 1 \\
\hline 125 & 1356 & Garfagnana & 1902-03-05 & 44.093 & 10.463 & 7.0 & 5.17 & 2 & 2 & & 1 & & \\
\hline 126 & 1384 & Marsica & 1904-02-24 & 42.100 & 13.320 & 8.5 & 5.67 & 8 & & 8 & & & \\
\hline 127 & 1420 & Calabria & 1905-09-08 & 38.670 & 16.070 & 11.0 & 7.06 & 60 & 33 & 33 & 23 & & 6 \\
\hline 128 & 1463 & Calabria meridionale & $1907-10-23$ & 38.130 & 16.020 & 8.5 & 5.93 & 12 & 12 & 6 & & & \\
\hline 129 & 1495 & Stretto di Messina & $1908-12-28$ & 38.150 & 15.680 & 11.0 & $7.24(10)$ & 58 & 47 & 25 & 5 & & 9 \\
\hline 120 & 1511 & Murlo & $1909-08-25$ & 43.150 & 11.403 & 7.5 & 5.40 & 1 & & & & & 1 \\
\hline 131 & 1555 & Area etnea & $1911-10-15$ & 37.700 & 15.150 & 10.0 & 5.28 & 8 & 1 & 6 & & 1 & 5 \\
\hline 132 & 1581 & Calabria settentrionale & $1913-06-28$ & 39.530 & 16.230 & 8.0 & 5.65 & 3 & 1 & 2 & & & \\
\hline 133 & 1596 & Tavernette & 1914-05-08 & 37.670 & 15.130 & 9.0 & 5.30 & 14 & 3 & 10 & & 5 & 5 \\
\hline 134 & 1604 & Area etnea & 1914-10-26 & 45.072 & 7.337 & 7.0 & 5.36 & 1 & 1 & & & & \\
\hline 135 & 1608 & Avezzano & 1915-01-13 & 42.013 & 13.530 & 11.0 & 6.99 & 66 & 41 & 38 & 12 & 3 & 9 \\
\hline
\end{tabular}


Table 1. Continued.

\begin{tabular}{|c|c|c|c|c|c|c|c|c|c|c|c|c|c|}
\hline $\begin{array}{l}\# \\
\text { eqk }\end{array}$ & $\begin{array}{l}\text { ID } \\
\text { CPTI04 }\end{array}$ & $\begin{array}{l}\text { Epicentral } \\
\text { area }\end{array}$ & Date & Lat $^{\circ}$ & Long $^{\circ}$ & Io(MCS) & $\begin{array}{l}M_{\mathrm{W}} \\
\text { (depth) }\end{array}$ & Sites & $\begin{array}{l}\text { Land- } \\
\text { slides }\end{array}$ & $\begin{array}{l}\text { Ground- } \\
\text { cracks }\end{array}$ & $\begin{array}{l}\text { Lique- } \\
\text { faction }\end{array}$ & $\begin{array}{l}\text { Surface } \\
\text { faulting }\end{array}$ & $\begin{array}{l}\text { Ground- } \\
\text { changes }\end{array}$ \\
\hline 136 & 1630 & Alto Adriatico & 1916-05-17 & 44.000 & 12.630 & 8.0 & 5.85 & 1 & & 1 & & & \\
\hline 137 & 1637 & Alto Adriatico & 1916-08-16 & 43.970 & 12.670 & 8.0 & 5.92 & 5 & & 3 & 7 & & 1 \\
\hline 138 & 1650 & Ternano & $1917-04-26$ & 43.465 & 12.125 & 9.0 & 5.80 & 4 & 1 & 3 & 3 & & \\
\hline 139 & 1651 & Monterchi-Citerna & $1917-05-12$ & 42.580 & 12.630 & 7.5 & 5.11 & 1 & 1 & & & & \\
\hline 140 & 1684 & Mugello & $1919-06-29$ & 43.950 & 11.480 & 9.0 & 6.18 & 7 & 3 & 5 & 4 & & \\
\hline 141 & 1687 & Piancastagnaio & 1919-09-10 & 42.793 & 11.788 & 8.0 & 5.38 & 1 & & & 1 & & \\
\hline 142 & 1708 & Garfagnana & 1920-09-07 & 44.180 & 10.280 & 9.5 & 6.48 & 23 & 25 & 6 & & & 1 \\
\hline 143 & 1800 & Colli Albani & $1927-12-26$ & 41.700 & 12.700 & 7.0 & 5.02 & 1 & 2 & & & & \\
\hline 144 & 1805 & Carnia & $1928-03-27$ & 46.372 & 12.975 & 8.5 & 5.75 & 17 & 26 & 4 & & & \\
\hline 145 & 1841 & Senigallia & $1930-07-23$ & 41.050 & 15.370 & 10.0 & $6.72(15)$ & 29 & 32 & 15 & & 4 & 2 \\
\hline 146 & 1847 & Irpinia & $1930-10-30$ & 43.659 & 13.331 & 9.0 & 5.94 & 2 & 1 & & 1 & & \\
\hline 147 & 1886 & Maiella & $1933-09-26$ & 42.050 & 14.180 & 8.5 & 5.68 & 2 & 2 & & & & \\
\hline 148 & 1921 & Bosco Cansiglio & 1936-10-18 & 46.088 & 12.380 & 9.0 & 5.90 & 6 & 2 & 5 & & & 1 \\
\hline 149 & 1950 & Garfagnana & $1939-10-15$ & 44.119 & 10.255 & 6.5 & 5.20 & 1 & 1 & & & & \\
\hline 150 & 1995 & Valle dello Staffora & $1945-06-29$ & 44.830 & 9.130 & 7.5 & 5.15 & 1 & 1 & & & & \\
\hline 151 & 2007 & Calabria centrale & $1947-05-11$ & 38.650 & 16.520 & 8.0 & 5.71 & 1 & 1 & & & & \\
\hline 152 & 2175 & Irpinia & $1962-08-21$ & 41.130 & 14.970 & 9.0 & 6.19 & 2 & 1 & & & 1 & 1 \\
\hline 153 & 2246 & Valle del Belice & 1968-01-15 & 37.770 & 12.980 & 10.0 & 6.12 & 15 & 7 & 9 & 7 & & \\
\hline 154 & 2294 & Tuscania & $1971-02-06$ & 42.442 & 11.846 & 7.5 & 4.90 & 2 & 2 & 1 & & & 1 \\
\hline 155 & 2363 & Friuli & 1976-05-06 & 46.241 & 13.119 & 9.5 & $6.43(06)$ & 103 & 84 & 17 & 46 & & 14 \\
\hline 156 & 2366 & Friuli & 1976-09-15 & 46.250 & 13.120 & 8.5 & $5.92(11)$ & 9 & 5 & 5 & 5 & & 2 \\
\hline 157 & 2400 & Valnerina & 1979-09-19 & 42.720 & 13.070 & 8.5 & $5.90(06)$ & 1 & 1 & & & & \\
\hline 158 & 2413 & Irpinia-Basilicata & $1980-11-23$ & 40.850 & 15.280 & 10.0 & $6.89(12)$ & 216 & 239 & 119 & 27 & 86 & 17 \\
\hline 159 & 2441 & Appennino Abruzzese & 1984-05-07 & 41.666 & 14.057 & 8.0 & $5.93(20)$ & 6 & 4 & 2 & & & \\
\hline 160 & 2478 & Sicilia sud-orientale & $1990-12-13$ & 37.266 & 15.121 & 7.0 & $5.68(07)$ & 1 & & & 2 & & \\
\hline 161 & 2515 & Umbria-Marche & 1997-09-26 & 43.019 & 12.879 & 8.5 & $6.05(10)$ & 203 & 194 & 82 & & 3 & 4 \\
\hline 162 & 2522 & Appennino Calabro-Lucano & 1998-09-09 & 40.038 & 15.937 & 6.5 & $5.68(29)$ & 51 & 56 & 5 & & 2 & \\
\hline 163 & 2546 & Tirreno meridionale & 2002-09-06 & 38.081 & 13.422 & 6.0 & $5.90(27)$ & 1 & 1 & 1 & & & \\
\hline 164 & 2550 & Molise & 2002-10-31 & 41.694 & 14.925 & 7.5 & $5.74(25)$ & 1 & 1 & 1 & 1 & & \\
\hline 165 & NA & L'Aquila & 2009-04-06 & 42.334 & 13.334 & 9.0 & $6.20(08)$ & 168 & 95 & 5 & 5 & 65 & 1 \\
\hline 166 & NA & Emilia & 2012-05-20 & 44.889 & 11.228 & 7.5 & $5.90(06)$ & 1343 & 12 & 16 & 1514 & & \\
\hline
\end{tabular}

Bold font: multiple events for which cumulative effects were likely.

the first mainshock (20 May) produced a greater number of ground effects than the second mainshock (29 May), although the two shocks had almost the same magnitude (5.9 vs. 5.8, respectively) and the same focal mechanism (reverse). One explanation could be the different focal depth, $6 \mathrm{~km}$ for the former and $10 \mathrm{~km}$ for the latter, although other differences, such as, amongst others, soil compaction after the first shock could have played a role in determining the lesser number of ground effects of the second shock.

In 1987, Zecchi published a distribution map of the geomorphologic effects induced by earthquakes that hit Italy before 1986. Unfortunately, the work did not include any database or table of the affected localities, but only a map available in a printed format. The distribution of earthquakes listed as triggering events is shown in Fig. 6 in terms of epicentral intensities, where, on the secondary $x$ axis, an approximate correspondence with moment magnitude is shown, based on a correlation provided by the CPTI earthquake catalogue's authors (Gruppo di Lavoro CPTI, 2004) and therefore specifically calibrated for Italian earthquakes. The threshold epicentral intensity of ground failures occurrence is the 6th degree of the Mercalli scale for both catalogues, corresponding to a moment magnitude 4.5-5. In this epicentral intensity class the CEDIT database reports a lesser number of earthquakes than the catalogue by Zecchi. The difference can be explained considering that the catalogue by Zecchi reported for the 6th degree of epicentral intensity only earthquakes that occurred prior to the instrumental age or were located offshore, for which successive studies have reassessed the real occurrence of ground failures triggered by those earthquakes.

The spatial distribution of the earthquakes and affected localities listed in the CEDIT database is shown in Fig. 7. The highest concentration is along the Apennine chain, with some relevant clusters associated with the most well-documented and recent events such as, from north to south, the 1976 Friuli $M_{\mathrm{w}}=6.4$ earthquake, 1997 Umbria-Marche $M_{\mathrm{W}}=6.0$ earthquake, 2009 L'Aquila $M_{\mathrm{w}}=6.2$ earthquake, 2012 Emilia $M_{\mathrm{w}}=5.9$ earthquake, 1980 Irpinia $M_{\mathrm{w}}=6.9$ earthquake and the earthquakes above $M_{\mathrm{w}}=6.5$ that hit the Calabria region in 1783,1905 and 1908.

The lithological features of localities affected by ground failures are shown in Fig. 8, based on the official Italian Geologic Map at scale 1:100000 (ISPRA, available online at http://sgi.isprambiente.it/geoportal/catalog/content/ project/litologica.page). Due to the map scale resolution, only localities reported in the most recent earthquakes (since 1908) for which a reliable location is available (error within $1 \mathrm{~km}$ ) are graphed. While the relative abundance of liquefaction in the class of alluvia and debris is obvious, landslides 


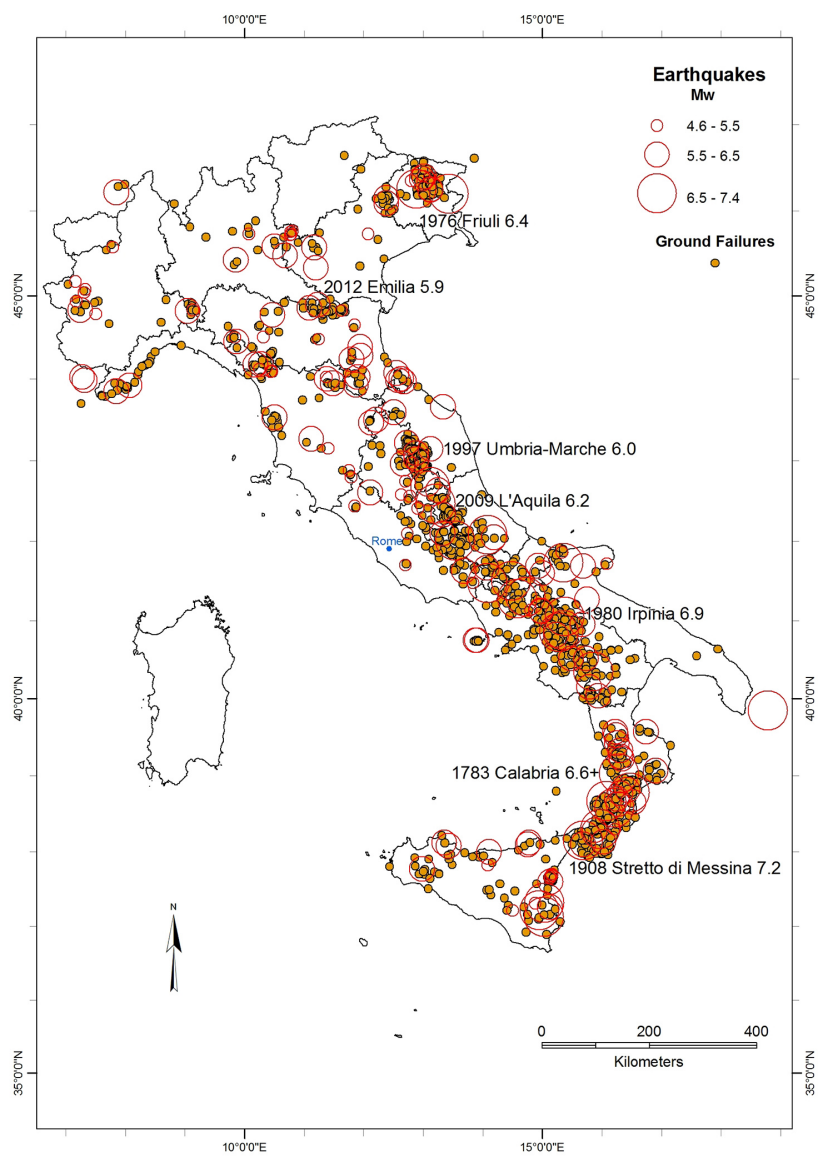

Fig. 7. Spatial distribution of earthquakes and localities affected by ground effects reported in the CEDIT database. Earthquakes that contributed most to the inventoried ground failures are marked on the map with the corresponding $M_{\mathrm{W}}$.

are most concentrated in limestone, due to the wide outcropping of calcareous rocks in the Apennines which represent the orogenic area with the highest seismicity in the Italian peninsula. This supports the idea that most of the undefined landslides of the CEDIT database can be ascribed to falls and disrupted slides (Keefer's type-1category).

It is worth noting that some irresolution still exists in the attribution of lithology to the ground effects (e.g. liquefaction effects reported in weak or hard rocks) due to the combined effect of the map scale resolution and of the basic information contained in the geological map that mainly refer to the substratum, rather than to the overlying quaternary deposits in which most of the ground failures usually occur.

\section{Relationships between ground failures and seismic parameters}

Earthquakes may trigger different types of ground failure depending on the released seismic energy, source-to-site distance and local conditions. As far as the source energy is con-

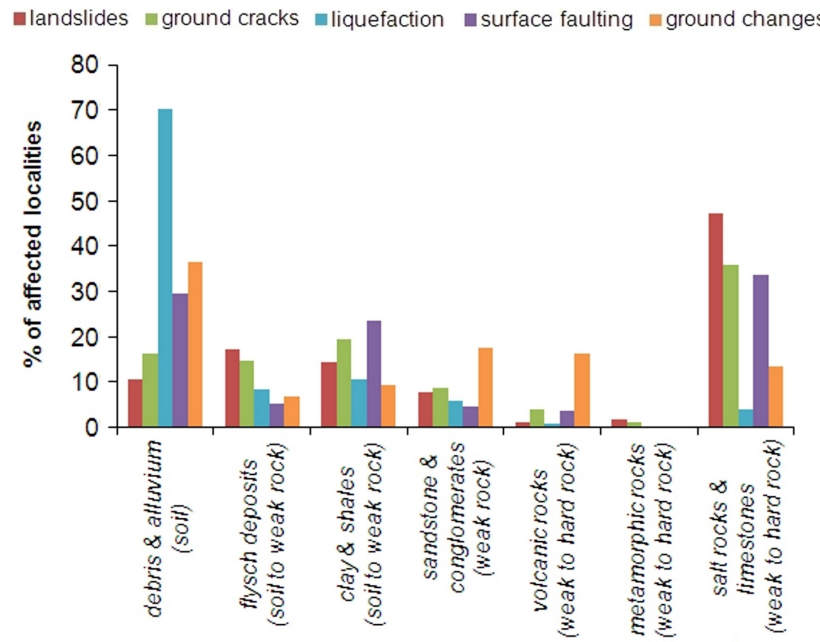

Fig. 8. Percentage of localities affected by ground failures in each lithological unit (ISPRA, 2004) for seismic events occurring since the 1908 Messina Strait earthquake. The lithotechnical attributes of each lithology are provided in brackets.

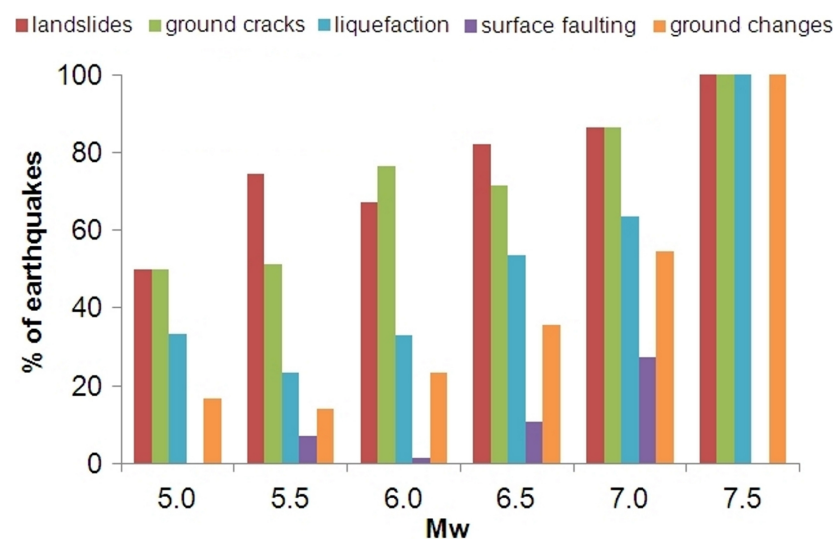

Fig. 9. Percentage of earthquakes in each magnitude class for which a specific type of ground failure was reported.

cerned, Fig. 9 shows the relative distribution of earthquakes per magnitude class for which a specific type of ground failure was reported. Landslides and ground cracks are reported in not less than $50 \%$ of earthquakes in each magnitude class, percentages that progressively increase up to $100 \%$ for earthquakes above magnitude 7 . The same trend can be observed for liquefaction and ground changes with a relative minimum at magnitude interval 5-5.5, due to the considerable increase in the number of earthquakes in this magnitude class relative to the previous class $\left(M_{\mathrm{w}}=4.5-5\right)$. The absence of earthquakes above magnitude 7 documenting the surface-faulting effect is because they occurred in times when the scientific community did not yet acknowledge such effects, whose systematic study and recognition started only with the most recent earthquakes, as also witnessed by Fig. 2. 


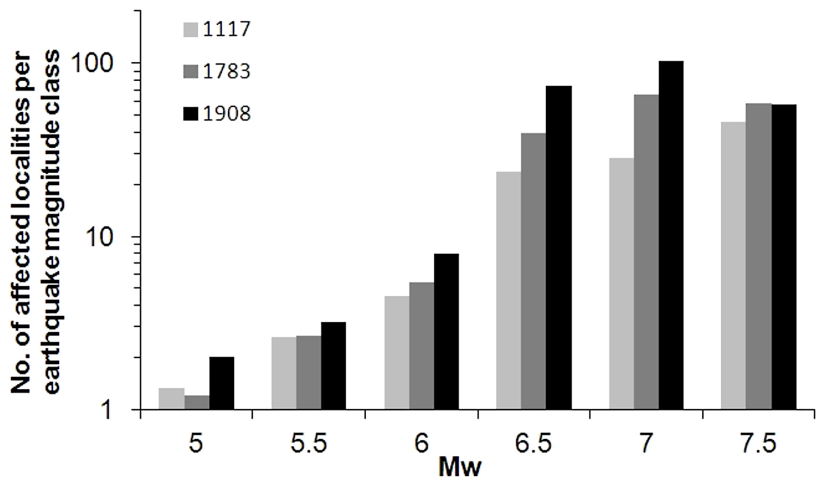

Fig. 10. Average number of localities affected by ground failures for each magnitude class and different time intervals related to the completeness of available information according to the time graph shown in Fig. 4.

The average number of affected localities for earthquakes in each magnitude class is shown in Fig. 10. Data have been disaggregated into time intervals referring to the key dates of increase in the completeness of documented information. The number of localities affected by ground effects continuously increases with the earthquake magnitude, with the shortest period (since 1908) being more complete than the other intervals. Only the last magnitude class $\left(M_{\mathrm{w}}=7-7.5\right)$ shows a lesser number of affected localities per earthquake magnitude since they all occurred before the availability of aerial surveys (the second half of 20th century) after that a more complete detection of ground failures is assured, even in the less populated areas.

Site intensity is a ground motion parameter that can be used to estimate the shaking level of a site on the basis of the effects (i.e. damage) locally produced by the earthquake. Site intensity is the only parameter that can be used to represent the local seismic shaking in historical earthquakes before the instrumental age. Nevertheless, site intensity is an integral parameter because it includes information about the shaking level (seismic demand) and the site response (seismic capacity). Distributions of surface faulting as a function of site intensity and distance are quite meaningless, as surface faulting is a primary effect being related only to the seismic source energy release. Therefore, surface faulting has been discarded from every correlation with site intensity and distance.

Figure 11 shows the number of earthquakes in which each ground-failure category was reported at a particular minimum intensity (Keefer, 1984). As far as the intensity scale is concerned, most of the site intensities described in the Italian earthquake catalogues use the Mercalli-Cancani-Sieberg scale (Sieberg, 1923), which strictly conforms, at least up to the 10th degree (Musson et al., 2010), to the Modified Mercalli intensity scale (Richter, 1958) and, ultimately, to the European Macroseismic Scale (Grünthal, 1998).

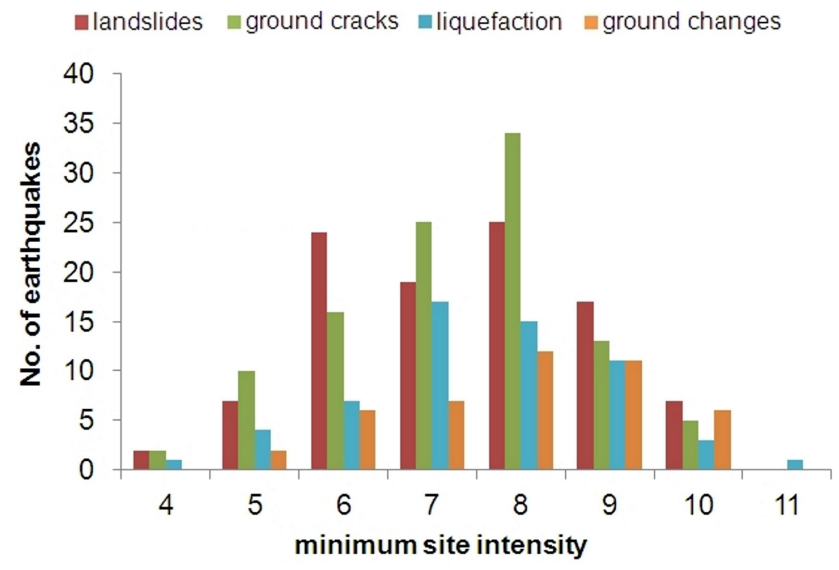

Fig. 11. Minimum site intensity at which ground failures occurred in the earthquakes listed in the CEDIT database.

Ground cracks are associated with the lowermost threshold intensity (4-5 on the MCS scale), followed by landslides with a threshold intensity between 5 and 6 , whereas the other ground failures display higher threshold intensities, such as 6-7 for liquefaction and 7-8 for ground changes. Threshold intensity is assumed to be a sudden increase in the number of earthquakes per minimum site intensity (a relative maximum in the first derivative).

Site intensity is assessed on the basis of the damage degree to buildings (EMS scale) or the ground effects (ESI scale) and on their relative abundance. Indeed, a weak motion can produce ground effects only in the soils that are already close to their failure state when the earthquake occurs, as opposed to a strong motion that can produce ground effects even in the soils that are quite stable in static conditions. Figure 12 shows the likelihood of a ground effect being observed given a site intensity that represents a relative measure of ground shaking. The absolute frequencies (shown as histograms) for each type of ground failure have been used to derive a probability curve according to the Weibull distribution, which is aimed to simulate the reaching of failure states. Looking, for instance, at ground cracks, it can be seen that the frequency distribution is apparently bimodal, given the relative maxima at site intensities 8 and 10. This makes all inferential statistics meaningless, whereas modelling these frequencies with a probability distribution makes feasible the comparison concerning the relative likelihood that each ground failure can be triggered at a given site intensity. Thus, at the lowermost site intensities ground cracks are more likely to be triggered than landslides, whereas liquefaction approaches landslides at the highest site intensities. The probability trends can be interpreted as measures of the progressive increase of the seismic shaking required to trigger ground failures: ground cracks initiate at the lowest seismic level intensities, and after they evolve at the stage of mass movements (landslides); as the seismic level increases again, pore water 


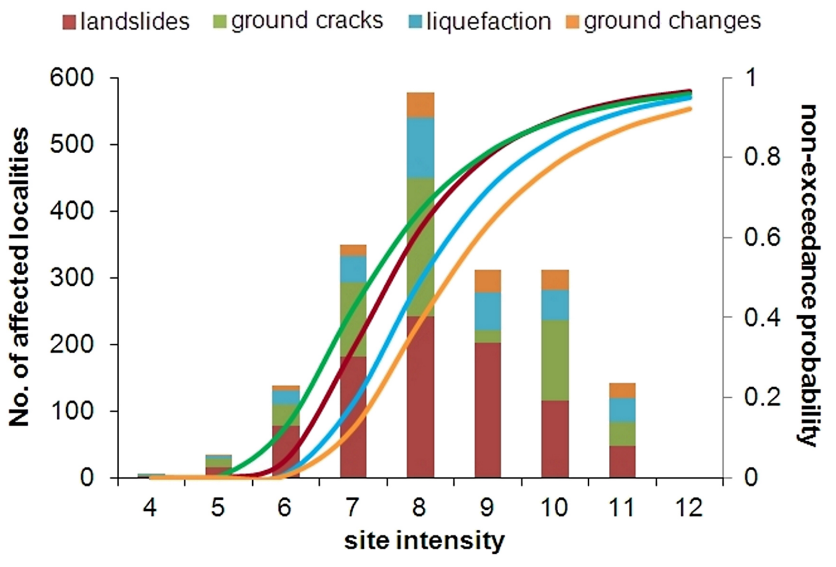

Fig. 12. Frequency distribution and non-exceedance probability (Weibull distribution) of site intensity for localities affected by ground effects.

overpressures generate and can progressively evolve into liquefaction, cyclic mobility and flow failures that at the ultimate stage can produce ground-level changes such as subsidence over wide areas.

Source-to-site distance is a key parameter for characterising the susceptibility of the ground to fail under the seismic shaking. In fact, distance modulates the seismic shaking by attenuating the amplitude and altering the duration and fundamental period of vibration, and all these factors influence the ground response. Thus, a relationship between the earthquake energy release and the distance at which ground failures can occur is straightforward.

Figure 13a shows the distribution of the maximum epicentral distance of landslides with the earthquake magnitude for the whole time span covered by the database (1117-2012). There is a great data scatter that makes any best-fit statistically meaningless; the most scattered data belong to earthquakes that occurred prior the instrumental age or even before the Calabria earthquakes of 1783. In fact, in his paper from 2002, Keefer reported that before 1783 “...historical accounts of the occurrence of landslides in earthquakes are typically so incomplete and vague that conclusions based on these accounts are of limited usefulness". According to this notice, Figure 13b shows the distribution limited to the time span 1783-2012. The reduction of the time span brings an evident improvement of the goodness of fit due to the greater accuracy of the observations. Taking also into account the greater precision gained after the advent of the instrumental age (Fig. 13c), the goodness of fit improves again: all the data, including error bars, are encompassed into one standard error of best fit, except for one rockfall that occurred during the 1928 Carnia (Northern Italy) $M_{\mathrm{w}}=5.8$ earthquake that indeed also lies outside Keefer's (1984) upper bound for falls and disrupted slides.

Figure 14 shows the magnitude-distance distribution for liquefaction. This effect shows a different pattern from land-
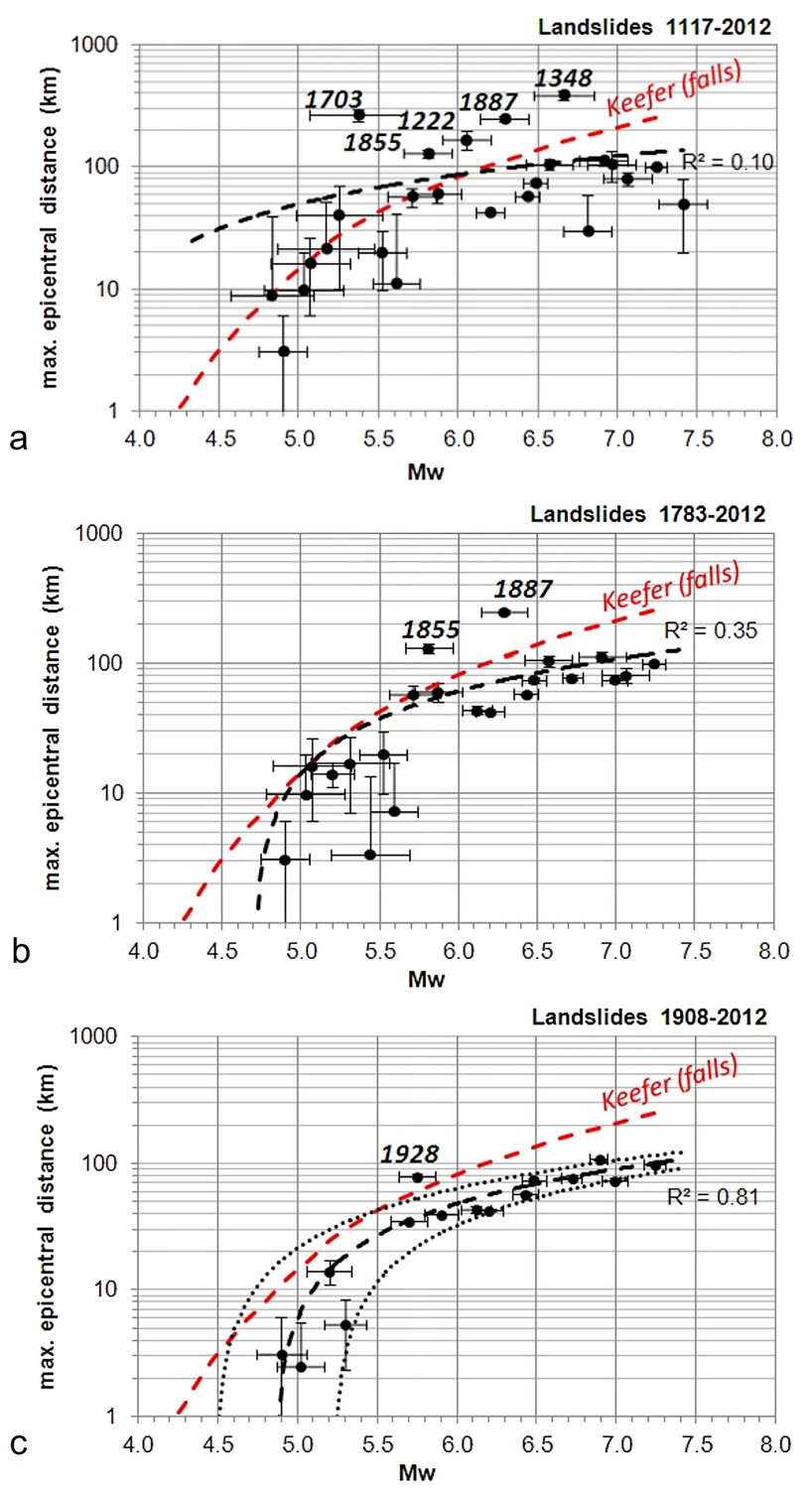

Fig. 13. Magnitude-distance relationships for landslides in different time periods (a: 1117-2012; b: 1783-2012; c: 1908-2012) compared to Keefer's (1984) upper bound for falls and disrupted slides (red dashed line). Black dotted lines in (c) refer to one standard error straddles the best-fit line (black dashed line).

slides. In fact, in this case the goodness of fit does not improve with the reduction of the time span, and the time interval 1908-2012 has too few data to be displayed. In fact, liquefaction of the Emilia 2012 earthquake lies above the bestfit line in the graph 1783-2012, whereas it is well captured by the best-fit line in the graph 1117-1783. Moreover, some outliers (shown as open circles) were discarded from the analysis, as they were documented in literature to be exceptional in terms of epicentral distance or as they were described with vague attributes in the available historical sources. This choice allowed also for the relationships to be non-flat or 

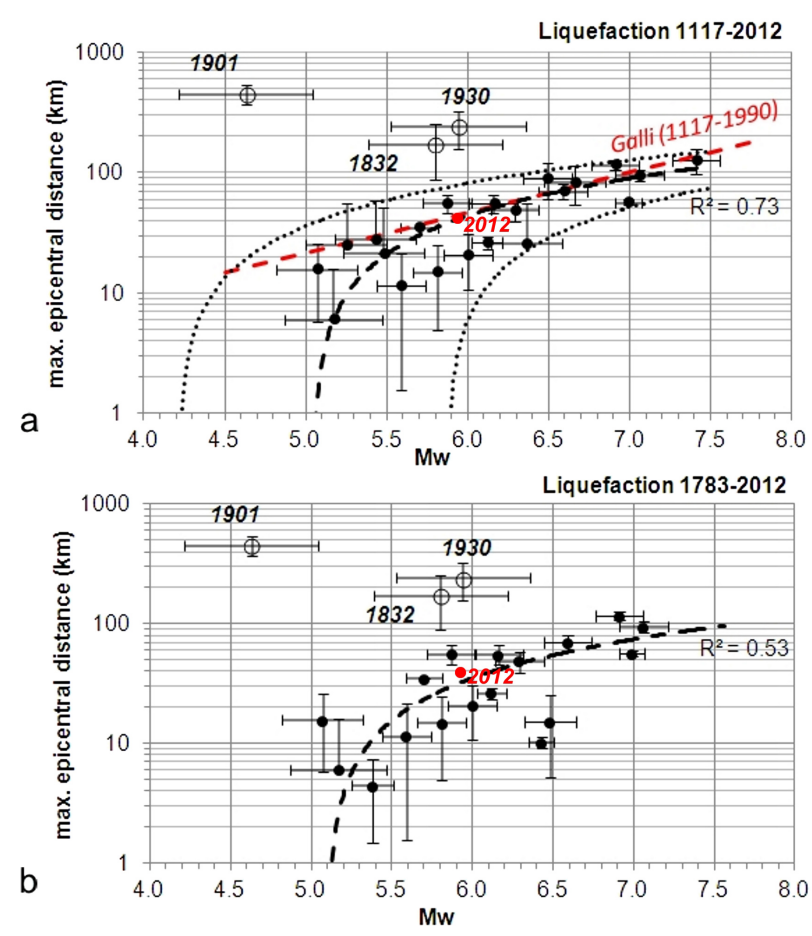

Fig. 14. Magnitude-distance relationships for liquefaction in different time periods (a: 1117-2012; b: 1783-2012). In (a) the comparison with the upper bound curve by Galli (2000), derived from Italian earthquakes that occurred in the period 1117-1990 is shown (red dashed line). Black dotted lines in (a) refer to two standard errors across the best-fit line (black dashed line). The red full circle corresponds to the liquefaction effects of the 2012 Emilia earthquake. Few outliers (black open circles) have resulted to be statistically meaningless and therefore they were discarded from the regression analysis.

even negative. Data distribution is more scattered than in the case of landslides and requires two standard errors to be encompassed (Fig. 15a). The comparison with the upper bound for liquefaction obtained by Galli (2000) for Italian earthquakes that occurred during 1117-1990 shows that it is not conservative for a significant $M_{\mathrm{w}}$-range (5.9 to 7). The main inference is that liquefaction needs more accurate investigations during earthquakes in order to be properly addressed and parameterised so that some conclusive relationships can be drawn.

\section{Discussion}

The CEDIT database represents a collection of information of the ground failures triggered by the most important earthquakes that hit in Italy in the last millennium. As for every collection of historical data, two main problems arise that need a thorough discussion in order to highlight advantages and shortcomings of such a kind of repository: data completeness and accuracy.

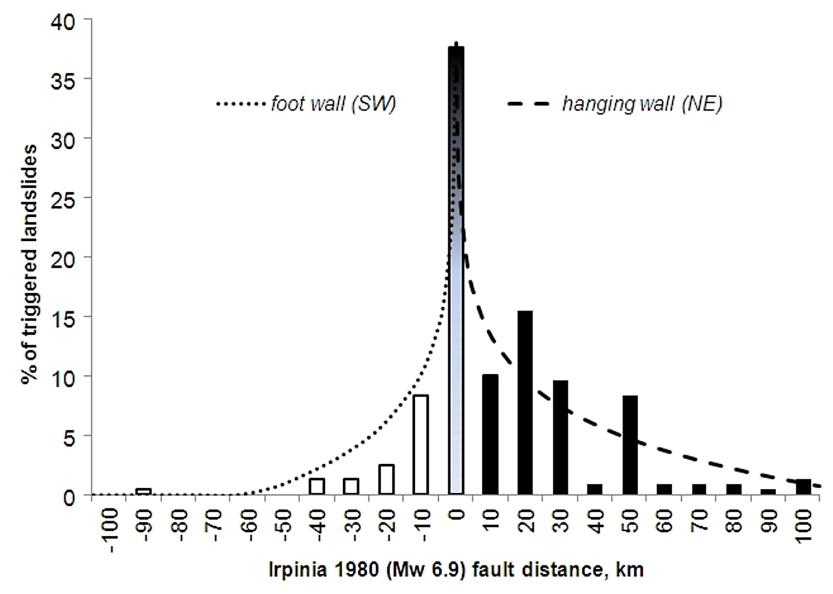

Fig. 15. Relative distribution of landslides as a function of the fault distance on the hanging wall and the foot wall of the 1980 Irpinia $M_{\mathrm{W}}=6.9$ earthquake main causative fault (normal faulting NE dipping).

Data incompleteness is an unavoidable problem when referring to historical or even pre-historical events. Nevertheless, even for current events data completeness can be problematic, due to the scale of the survey and the size of the detectable effects (Harp et al., 2011). As an example, this is the case for the different liquefaction inventories compiled after the recent Emilia 2012 earthquake in northern Italy and reported by Emergeo Working Group (2013), Di Manna et al. (2012) and the Liquefaction Working Group of the Emilia-Romagna Regional Government (http://ambiente.regione.emilia-romagna.it/geologia/ temi/sismica/liquefazione-gruppo-di-lavoro). The effect of incompleteness is particularly meaningful when dealing with the computation of the frequency of occurrence, as it is the case for the computation of seismicity rates in earthquake hazard analysis, but, where this is not the case, it is more important to be confident that the most important cases for which some documentation is available have been captured. In fact, the detection of extreme events such as large-distance effects from the earthquake sources, can provide the rationale for the development of scenarios at regional scale, even if a formal assessment of the ground failure hazard is prevented by the data incompleteness. Moreover, the availability of even a few instances of ground failures in previous earthquakes are useful for a perspective assessment of the environmental response to earthquakes, as is the case of the Emilia 2012 earthquake that showed a similar pattern of ground failures as reported and documented by the CEDIT catalogue for a previous earthquake that occurred in the same area in 1570.

As far as the accuracy of the earthquake and ground effect parameters determination is concerned, uncertainty affects to a varying extent all the parameters, characterising both the triggering events and the triggered effects and encompassing 
errors from the epicentral coordinates and magnitude of the causative earthquakes, to the site coordinates of the induced ground effects.

Epicentral errors as large as hundreds of kilometres were reported by Postpischl (1985a, b) for the PFG (Italian Geodynamics Project) earthquake catalogue compiled on the basis of previous historical catalogues (Bonito 1691, Baratta 1901, Cavasino 1931-1935, Iaccarino 1968-1971, Carrozzo 1973, Peronaci 1973, ENEL 1979). In 1990s the Italian seismological community launched a program to provide a reliable earthquake catalogue for the seismic hazard map of Italy, resulting in the NT4.1 earthquake catalogue (1997) and the following CPTI series of earthquake catalogues (1999, 2004 and 2011: http://emidius.mi.ingv.it/CPTI/). Starting from the former CPTI99 catalogue, historical epicentres were determined using the Boxer method by Gasperini et al. (1999). Nevertheless, a formal error for macroseismic determination of epicentral coordinates cannot be computed, as it depends, from time to time, on the number of felt points and on their azimuth and spatial distribution. The uncertainty associated with the algorithm used for the epicentral location provides only a measure of the reliability of the estimate: for instance, a large value may only implicitly indicate the existence of anomalous intensity points (as in the case of offshore epicentres) or incompleteness of data distribution, for instance sparsely populated areas (see Gasperini et al., 1999: Appendix 1). The same effects apply to the uncertainty in the magnitude assessment of historical (pre-instrumental) earthquakes, since the severity of the earthquake is dependent on the mesoseismal area that, in turn, is dependent on the felt points (which affects the epicentral location, too). A crude estimate of the relationship between error and magnitude value, as derived from the CPTI catalogue, shows how error tends to be inversely proportional to the magnitude value and also progressively decreases with time toward the present, due to the increase of macroseismic information.

The uncertainty in the distance computation of the ground effect from the earthquake source is in turn a joint function of the error in the epicentral location and the error in the ground effect location. Since the former is difficult to quantify, only the latter can be reasonably assessed considering the timing and the toponym of localities where ground effects are reported in historical chronicles. According to the administrative hierarchy of Italian territories, the CEDIT database assigns an error to each ground effect location based on the spatial extent of the place name according to the following ranking scheme, from closer to farther:

1. Site (GPS measurement): no error or negligible.

2. Village (area extent of square kilometres): average error $1 \mathrm{~km}$.

3. Town (area extent of tens of square kilometres): average error $3 \mathrm{~km}$.
4. City (area extent of hundreds of square kilometres): average error $10 \mathrm{~km}$

5. Province (area extent of thousands of square kilometres, comparable to US county or England shire): average error $30 \mathrm{~km}$.

The older the effect, the higher the error: for instance, site error is assigned only to ground effects detected since the 1990s, when systematic detection of ground effects through GPS measurements became the practice.

In this direction, the greater availability of good quality data for the most recent earthquakes, makes it possible to infer more accurate information on the spatial distribution of ground effects. For instance, landslides triggered by the 1980 Irpinia earthquake (Fig. 15) were more widespread on the hanging wall of the causative fault (normal faulting NE dipping) than on the footwall, as a result of the activation of another fault antithetic to the main one (Westaway and Jackson, 1984; Bernard and Zollo, 1989).

This is only an example of the potential of the CEDITDB to investigate more in depth the relationships between triggering events and induced effects. Additional and more accurate relationships can be inferred from the spatial distribution of ground effects and ground motion parameters deriving from the seismic records.

\section{Conclusions}

This paper describes the new release of the CEDIT catalogue, a database of information regarding localities affected by ground failures triggered by the strongest earthquakes that have occurred in Italy over the last millennium. The database is an update of the former version released at the end of the 1990s, and extends the investigated time period and includes some specific studies on past earthquakes with improved descriptions of their ground effects. The database has been available online since January 2013 at http://www.ceri.uniroma1.it/cn/gis.jsp.

The distribution of earthquake magnitudes indicates that a threshold value of approximately 4.5 is likely to be the minimum magnitude required to trigger ground failures.

The rate of increase of ground failures with the increase in seismic energy release (i.e. with earthquake magnitude) clearly indicates that the ground effects requiring stronger energy contents, such as liquefaction, ground changes and surface faulting, increase much more with the earthquake magnitude than landslides and ground cracks, which can also be triggered by seismic events of lower energy.

Local site intensity is used to account for the seismic shaking responsible for the triggering of many of the ground failures, as most of the reported effects occurred in historical earthquakes prior to the advent of monitoring systems. The threshold intensity progressively increases from landslides 
and ground cracks (5-6 MCS) to liquefaction (6-7 MCS) and ground changes (7-8 MCS).

Magnitude-distance relationships for landslides are more reliably drawn than for liquefaction, whose effects appear to be more scattered. Such relationships, when compared with similar ones from literature, are shown to be more or less conservative depending on the time span considered. For instance, the accuracy of the most recent data on landslides leads to more reliable relationships that can be usefully applied for seismic landslide scenarios over large areas. At the same time, the completeness of the inventories for the most recent events contribute to reducing the scatter of the inferred relationships, and so increasing their precision.

One shortcoming when using ground failures as a proxy for the seismic shaking or, conversely, for magnitudedistance relationships, is that they depend on the properties of the lithologies when the feature formed (e.g. relative density, degree of saturation, boundary stress conditions, joint orientation, and many others), and for this reason the data are widely scattered and the resulting relationships are affected by high uncertainty.

Until now, there has been a lack of databases containing data on ground failures caused by earthquakes and a comprehensive analysis of the relationships between ground effects as well as ground motion. The main reason for this lack is the scarcity of ground-motion records and the mismatch between the location of sites affected by ground failures and recording stations. Nevertheless, the recent increase of availability of high-quality ground-motion records make it possible in some instances to tentatively approach this important target. In Italy, for instance, the good coverage of strong motion recording stations, available since the 1976 Friuli $M_{\mathrm{w}}=6.4$ earthquake, would make it possible to investigate the relationships between ground shaking and the triggered ground effects, deriving the ground motion at localities affected by ground failures either developing earthquake-specific ground motion attenuation relationships or using interpolation techniques available in many computer programs of geostatistics.

Acknowledgements. The authors wish to thank C. Fortunato for her contribution on data cataloguing and A. Fantini, F. Nardoni and P. Sarandrea for mastering the online version of the CEDIT-DB. The authors also thank W. Murphy and one anonymous reviewer for their useful suggestions and the editorial comments and reviewing that contributed to significantly improving the manuscript.

Edited by: D. Keefer

Reviewed by: W. Murphy and one anonymous referee

\section{References}

Alfaro, P., Delgado, J., García-Tortosa, F. J., Giner, J. J., Lenti, L., López-Casado, C., Martino, S., and Scarascia Mugnozza, G.: The role of near-field interaction between seismic waves and slope on the triggering of a rockslide at Lorca (SE Spain), Nat. Hazards Earth Syst. Sci., 12, 3631-3643, doi:10.5194/nhess-123631-2012, 2012.

Allen, T. I., Marano, K., Earle, P. S., and Wald, D. J.: PAGER-CAT: A composite earthquake catalog for calibrating global fatality models, Seism. Res. Lett., 80, 50-56, 2009.

Bernard, P. and Zollo, A.: The Irpinia (Italy) 1980 earthquake: Detailed analysis of a complex normal faulting, J. Geophys. Res.Sol. Ea., 94, 1631-1647, 1989.

Bird, J. F. and Bommer, J. J.: Earthquake losses due to ground failure, Eng. Geol., 75, 147-179, 2004.

Bommer, J. J. and Rodríguez, C. E.: Earthquake-induced landslides in Central America, Eng. Geol., 63 , 189-220, 2002.

Bozzano, F., Martino, S., Naso, G., Prestininzi, A., Romeo, R. W., and Scarascia Mugnozza, G.: The large Salcito landslide triggered by the 31st October 2002, Molise earthquake, Earthq Spectra, 20, 1-11, 2004.

Bozzano, F., Lenti, L., Martino, S., Paciello, A., and Scarascia Mugnozza, G.: Self-excitation process due to local seismic amplification responsible for the reactivation of the Salcito landslide (Italy) on 31 October 2002, J. Geophys. Res., 113, B10312, doi:10.1029/2007JB005309, 2008.

Bozzano, F., Lenti, L., Martino, S., Paciello, A., and Scarascia Mugnozza, G.: Evidences of landslide earthquake triggering due to self-excitation process, Int. J. Earth Sci., 100, 861-879, doi:10.1007/s00531-010-0514-5, 2011.

Camassi, R. and Stucchi, M.: Gruppo Nazionale per la Difesa dai Terremoti NT4.1, un catalogo parametrico di terremoti di area italiana al di sopra della soglia del danno. A parametric catalogue of damaging earthquakes in the Italian area. versione NT4.1.1 luglio 1997, http://emidius.mi.ingv.it/NT/CONSNT.html, 1997.

Carraro, F., Cavallin, A., Frascari, F., Gasperi, G., Gelmini, R., Govi, M., Manfredini, U., Martinis, B., Panizza, M., Petrucci, F., Stefanini, S., and Zanferrari, A.: The Friuli earthquake of May 6 , 1976 geology, Bollettino di Geofisica Teorica ed Applicata, 19, 755-808, 1976.

DBMI04: Database Macrosismico Italiano, INGV http://emidius. mi.ingv.it/DBMI04, 2004.

De Lorenzo, A. M.: Scilla inondata dal mare nella notte dopo il 5 febbraio 1783. In Memorie da servire alla storia sacra e civile di Reggio e delle Calabrie, Cronache e Documenti rari, 4 (4-7), Reggio Emilia (IT), 317-376, 1877.

Delfino, L. and Romeo, R. W.: C.E.D.I.T., Catalogo nazionale degli Effetti Deformativi del suolo Indotti da forti Terremoti, RAPPORTO TECNICO SSN/RT/97/04, 1997.

Delgado, J., Garrido, J., López-Casado, C., Martino, S., and Peláez, J. A.: On far field occurrence of seismically induced landslides, Eng. Geol., 123, 204-213, doi:10.1016/j.enggeo.2011.08.002, 2011.

Di Manna, P., Guerrieri, L., Piccardi, L., Vittori E., Castaldini, D., Bersulsconi, A., Bonadeo, L., Comerci, V., Ferrario, F., Gambillara, R., Livio, F., Lucarini, M., and Michetti A. M.: Ground effects induced by the 2012 seismic sequence in Emilia: implications for seismic hazard assessment in the Po Plain, Ann. Geo- 
phys., 55, 697-703, 2012,

http://www.ann-geophys.net/55/697/2012/.

DISS Working Group: Database of Individual Seismogenic Sources (DISS), Version 3.1.1: A compilation of potential sources for earthquakes larger than M 5.5 in Italy and surrounding areas, http://diss.rm.ingv.it/diss/, 2010.

Emergeo Working Group: Liquefaction phenomena associated with the Emilia earthquake sequence of May-June 2012 (Northern Italy), Nat. Hazards Earth Syst. Sci., 13, 935-947, doi:10.5194/nhess-13-935-2013, 2013.

Fortunato, C., Martino, S., Prestininzi, A., and Romeo, R. W.: New release of the Italian catalogue of earthquake-induced ground failures (CEDIT), Italian J. Eng. Geol. Environ., 2, 63-74, 2012.

Galli, P.: New empirical relationships between magnitude and distance for liquefaction, Tectonophysics, 32, 169-187, 2000.

Gasperini, P., Bernardini, F., Valensise, G., and Boschi, E.: Defining seismogeneic sources from historical earthquakes felt reports, B. Seismol. Soc. Am., 89, 94-110, 1999.

Gorum, T., Fan, X., van Westen, C. J., Huang, R. Q., Xu, Q., Tang, C., and Wang, G.: Distribution pattern of earthquake-induced landslides triggered by the 12 May 2008 Wenchuan earthquake, Geomorphology, 133, 152-167, 2008.

Graziani, L., Maramai, A., and Tinti, S.: A revision of the 17831784 Calabrian (southern Italy) tsunamis, Nat. Hazards Earth Syst. Sci., 6, 1053-1060, doi:10.5194/nhess-6-1053-2006, 2006.

Grünthal, G. (Ed.): European Macroseismic Scale 1998, Conseil de l'Europe, Cahiers du Centre Europèen de Gèodynamique et de Seismologie, vol. 15, 101 pp., 1998.

Grünthal, G. and Wahlström, R.: The European-Mediterranean Earthquake Catalogue (EMEC) for the last millennium, J. Seismol., 16, 535-570, 2012.

Gruppo di Lavoro CPTI: Catalogo Parametrico dei Terremoti Italiani, versione 2004 (CPTI04), INGV, Bologna, http://emidius.mi. ingv.it/CPTI04/, 2004.

Guerrieri, L., Porfido, S., Esposito, E., Blumetti, A. M., Michetti, A. M., and Vittori, E.: Cataloguing Earthquake Environmental Effects: a tool for the comparison of recent historical and paleoearthquakes, Proc. 1st INQUA-IGCP-567 International Workshop on Earthquake Archaeology and paleoseismology, Baelo Claudia, Spain, 2009.

Harp, E. L., Keefer, D. K., Sato, H. P., and Yagi, H.: Landslide inventories: The essential part of seismic landslide hazard analyses, Eng. Geol., 122, 9-21, 2011.

Hoyois, P., Below, R., Scheuren, J. M., and Guha-Sapir, D.: Annual disaster statistical review: numbers and trends 2006, Centre for Research on the Epidemiology of Disasters, School of Public Health, Catholic University of Louvain, 54 pp., 2007.

Hsieh, S. U. and Lee, C. T. : Empirical estimation of the Newmark displacement from the Arias intensity and critical acceleration, Eng. Geol., 122, 34-42, 2011.

I.N.G.: Catalogo dei forti terremoti in Italia dal 461 a.C. al 1980, edited by: Boschi E., Ferrari G., Gasperini P., Guidoboni E., Smriglio, G., and Valensise, G., ING-SGA, Bologna, 970 pp., 1995.

ISPRA: Carta Litotecnica d'Italia 1:100,000. Dipartimento Difesa del Suolo, available available at: http://sgi.isprambiente.it/ geoportal/catalog/content/project/litologica.page, 2004.

Jibson, R. W.: Regression models for estimating coseismic landslide displacement, Eng. Geol., 91, 209-218, 2007.
Jibson, R. W. and Harp, E. L.: Extraordinary Distance Limits of Landslides Triggered by the 2011 Mineral, Virginia, Earthquake, BSSA, 102, 2368-2377, doi:10.1785/0120120055, 2012.

Jibson, R. W., Harp, E. L., and Michael, J.,M.: A method for producing digital probabilistic seismic landslide hazard maps: an example from the Los Angeles, California area, US Geological Survey Open-File Report, 98-113, 1998.

Keefer, D. K.: Landslides caused by earthquakes, Geol. Soc. Am. Bull., 95, 406-421, 1984.

Keefer, D. K.: Investigating landslides caused by earthquakes - A historical Review, Surv. Geohys., 23, 473-510, 2002.

Kirschbaum, D. B., Adler, R., Hong, Y., Hill, S., and Lerner-Lam, A.: A global landslide catalog for hazard applications: method, results, and limitations, Natural Hazards, 52, 561-575, 2010.

Mancini, B., Martino, S., Prestininzi, A., Rischia, I., and Romeo, W. R.: Studio delle condizioni di stabilità dei versanti a seguito di forti terremoti: applicazione ad un'area tipica dell'Appennino Centrale, Mem. Soc. Geol. It., 56, 83-98, 2001.

Martini, A.: Manuale di metrologia, Ed. Loescher, Torino, 1883.

Martino, S., Prestininzi, A., and Scarascia Mugnozza, G.: Geological-evolutionary model of a gravity-induced slope deformation in the carbonate Central Apennines (Italy), Q. J. Eng. Geol. Hydrogeol., 37, 31-47, 2004.

Meletti, C., Patacca, E., and Scandone, P.: Construction of a seismotectonic model: the case of Italy, Pure Appl. Geophys., 157, $11-35,2000$.

Michetti, A. M, Esposito, E., Gurpinar, A., Mohammadioun, B., Mohammadioun, J., Porfido, S., Rogozhin, E., Serva, L., Tatevossian, R., Vittori, E., Audemard, F., Comerci, V., Marco, S., Mccalpin, J., and Morner, N. A.: The INQUA Scale. An innovative approach for assessing earthquake intensities based on seismically-induced ground effects in natural environment Memorie descrittive della Carta Geologica d'Italia, 67, 2004.

Michetti, A. M., Esposito, E., Guerrieri, L., Porfido, S., Serva, L., Tatevossian, R. Vittori, E. Audemard, F., Azuma, T., Clague, J., Comerci, V., Gürpinar, A., Mc Calpin, J., Mohammadioun, B., Mörner, N. A., Ota, Y., and Roghozin, E: Environmental Seismic Intensity scale - ESI 2007 La scala di Intensità Sismica basata sugli effetti ambientali - ESI 2007, Memorie descrittive della carta geologica D'Italia, LXXIV, APAT, 2007.

Minasi, A.: "Continuazione ed appendice sopra i tremuoti descritti nella relazione colla data di Scilla de 30 settembre 1783, con altro che accadde in progresso, Messina", 1785.

Musson, R. M. W., Grünthal, G., and Stucchi, M.: The comparison of macroseismic intensity scales, J. Seismol., 14, 413-428, 2010.

Petley, D.N., Dunning, S.A. and Rosser N.J.: The analysis of global landslide risk through the creation of a database of worldwide landslide fatalities, in: Landslide risk management, edited by: Hungr, O., Fell, R., Counture, R., and Ebergardt, E., Balkema, Amsterdam, 367-374, 2005.

Porfido, S., Esposito, E., Guerrieri, L., Vittori, E., Tranfaglia, G., and Pece, R.: Seismically induced ground effects of the 1805, 1930 and 1980 earthquakes in the Southern Apennines, Italy, Boll. Soc. Geol. It., 126, 333-346, 2007.

Postpischl, D.: Atlas of isoseismal Maps of italian Earthquakes, CNR-PFG, Quaderni de "La Ricerca Scientifica", 114, 2A, Bologna, 1985a. 
Postpischl, D.: Catalogo dei forti terremoti italiani dall'anno 1000 al 1980, CNR-PFG, Quaderni de "La Ricerca Scientifica", 114, 2B, Bologna, 1985b.

Prestininzi, A.: Il ruolo degli eventi naturali sulla evoluzione urbana del centro abitato di Caulonia-Castelvetere (Reggio Calabria), Geologia Applicata ed Idrogeologia, 30-1, 393-405, 1995.

Prestininzi, A. and Romeo, R.: Earthquake-induced ground failures in Italy, Eng. Geol., 58, 387-397, 2000.

Richter, C. F.: Elementary seismology, Freeman, San Francisco, 1958.

Rodriguez, C. E., Bommer, J. J., and Chandler, R. J.: Earthquakeinduced landslides: 1980-1997, Soil Dynam. Earthq. Eng., 18, 325-346, 1999.

Romeo, W. R.: Seismically induced landslide displacements: a predictive model, Eng. Geol., 58, 337-351, 2000.

Romeo R. W.: Emilia (Italy) M 5.9 earthquake on 20 May 2012: an unusual pattern of liquefaction, Ital. J. Eng. Geol. Environ., 2, 63-74, 2012.

Rovida, A., Camassi, R., Gasperini, P., and Stucchi, M.: CPTI11, the 2011 version of the Parametric Catalogue of Italian Earthquakes, edited by: Rovida, A., Camassi, R., Gasperini, P., and Stucchi, M., Milano, Bologna, http://emidius.mi.ingv.it/CPTI, 2011.

Sarconi, M.: Historia dé fenomeni del tremuoto avvenuto nella Calabria e nel Valdemone nell'anno 1783, Posti in luce alla Reale Accademia delle Scienze e delle Belle Lettere di Napoli, 1784.

Sepulveda, S. A., Murphy, W., Jibson, R. W., and Petley, D. N.: Seismically induced rock slope failures resulting from topographic amplification of strong ground motions: The case of $\mathrm{Pa}-$ coima Canyon, California”, Eng. Geol., 80, 336- 348, 2005.

Serva, L., Esposito, E., Guerrieri, L., Porfido, S., Vittori, E., and Comerci, V.: Environmental effects from five historical earthquakes in southern Apennines (Italy) and macroseismic intensity assessment: Contribution to INQUA EEE Scale Project, Quatern. Int., 173-174, 30-44, 2007.

Sieberg, A.: Geologische, physikalische und angewandte Erdbebenkunde, G. Fischer, Jena, 1923.

Sipkin, S. A., Person, W. J., and Presgrave, B. W.: Earthquake bulletins and catalogs at the USGS National Earthquake Information Center, IRIS Newsletter, 2000, 2-4, 2000.
Stucchi, M., Camassi, R., Rovida, A., Locati, M., Ercolani, E., Meletti, C., Migliavacca, P., Bernardini, F., and Azzaro, R.: DBMI04, il database delle osservazioni macrosismiche dei terremoti italiani utilizzate per la compilazione del catalogo parametrico CPTI04, Quaderni di Geofisica, 49, 38 pp., http://emidius. mi.ingv.it/DBMI04, 2007.

Tang, C., Zhu, J., Qi, X., and Ding, J.: Landslides induced by the Wenchuan earthquake and the subsequent strong rainfall event: A case study in the Beichuan area of China, Eng. Geol., 122, 22-33, doi:10.1016/j.enggeo.2011.03.013, 2011.

Tinti, S., Maramai, A., and Graziani, L.: The Italian Tsunami Catalogue (ITC), Version 2.0, avaiilable at: http://roma2.rm.ingv.it/ en/facilities/data_bases/27/catalogue_of_the_italian_tsunamis, 2007.

Tosatti, G., Castaldini, D., Barbieri, M., D’Amato Avanzi, G., Giannecchini, R., Mandrone, G., Pellegrini, M., Perego, S., Puccinelli, A., Romeo, R. W., and Tellini, C.: Additional Causes of Seismically-Related Landslides in the Northern Apennines, Italy, Revista de geomorfologie, 10, 5-21, 2008.

Utsu, T.: A list of deadly earthquakes in the World: 1500-2000, in: International handbook of earthquake engineering and seismology, edited by: Lee, W. K., Kanamori, H., Jennings, P. C., and Kisslinger, C., Amsterdam, Academic Press, 691-717, 2002.

Vivenzio, G.: "Historia de' Tremuoti avvenuti nella Provincia della Calabria ulteriore e nella Città di Messina nell'anno 1783 e di quanto nella Calabria fu fatto per lo suo risorgimento fino al 1787 preceduta da una Teoria”, Istoria Gen. De' Tremuoti, Vol. 1-2 Stamperia reale, Napoli, 1788.

Westaway, R. and Jackson, J. A.: Surface faulting in the southern Italian Campania-Basilicata earthquake of 23 Nov. 1980, Nature, 312, 436-438, 1984.

Youd, T. L. and Hoose, S. N.: Historic Ground Failures in Northern California Associated with Earthquakes: US Geological Survey Professional Paper 993, 177 pp. + 5 plates, 1978.

Zecchi, R.: Carta della distribuzione degli effetti geomorfologici indotti dai terremoti che hanno interessato l'Italia dall'anno 0 al 1986, Mem. Soc. Geol. It., 37, 823-826, 1987. 